\title{
Anti de Sitter quantum field theory and a new class of hypergeometric identities
}

\author{
Jacques Bros ${ }^{1}$, Henri Epstein ${ }^{2}$, Michel Gaudin ${ }^{1}$, Ugo Moschella ${ }^{3,4}$, Vincent Pasquier ${ }^{1}$ \\ ${ }^{1}$ Institut de Physique Théorique, CEA - Saclay, France \\ ${ }^{2}$ Institut des Hautes Études Scientifiques, 91440 Bures-sur-Yvette \\ ${ }^{3}$ Università dell'Insubria, 22100 Como, Italia, \\ ${ }^{4}$ INFN, Sez. di Milano, Italia
}

June 8, 2018

\begin{abstract}
We use Anti-de Sitter quantum field theory to prove a new class of identities between hypergeometric functions related to the Källén-Lehmann representation of products of two Anti-de Sitter two-point functions. A rich mathematical structure emerges. We apply our results to study the decay of unstable Anti-de Sitter particles. The total amplitude is in this case finite and Anti-de Sitter invariant.
\end{abstract}

\section{Introduction}

The interest in the Anti-de Sitter geometry and the corresponding classical and quantum field theories has gradually increased in recent years and gained an important place in theoretical physics. Today, studies in Anti-de Sitter field theory or researches using Anti-de Sitter techniques to compute amplitudes in other kind of (realistic) quantum field theories such as quantum chromodynamics play a central role in high energy physics.

Anti-de Sitter provides indeed access to nontrivial Minkowski quantum field theories in two ways. Through the Maldacena duality [1], Anti-de Sitter models correspond to conformal quantum field theories on the boundary. In this approach quantum theories on the Minkowski spacetime come from (and actually are believed to be equivalent to) models in higher dimensional Anti-de Sitter universes.

On the other hand, the Anti-de Sitter manifold may be also viewed as an infrared (covariant) regularization of the Minkowski spacetime [2]; Poincaré invariant models can be constructed by taking the flat limit of Anti-de Sitter ones. In this way one can gain information on Minkowskian quantum field theories from Anti-de Sitter models having the same spacetime dimensionality.

In both cases, the correspondences between Anti-de Sitter and Minkowski theories may be used to uncover new pieces of mathematics. The idea is that to a known relation existing on the Minkowski spacetime there should correspond a possibly unknown relation on the Anti-de Sitter universe and vicecersa.

In this paper we use this idea to guess and prove a new class of linearization identities among hypergeometric functions. This was suggested by a series of related papers [3, 4, 5] where we have considered particle decays in the de Sitter universe. The effort necessary to compute the Källén-Lehmann weights needed to evaluate the lifetime of de Sitter particles unveiled there a rich mathematical structure; new integral formulae for products of three Legendre functions followed.

Trying to solve the same problem in the Anti-de Sitter case provides a new class of nontrivial identities between hypergeometric functions. The mathematics behind these new identities is however quite different. 
In the end of the paper, as an application of our results, we briefly discuss the problem of particle decay in the Anti-de Sitter universe and its flat limit. This example will make clear the value of the Anti-de Sitter universe as an infrared regulator of calculations which are divergent in the flat case. In particular we compute the total probability of decay of a given Anti-de Sitter one-particle state into all possible two-particle states at first order in perturbation theory. This quantity is divergent both in the Minkowski and the de Sitter universes while it is perfectly finite and can be explicitly computed in the Anti-de Sitter case. This quantity, once it is divided by the radius of the Anti-de Sitter universe, has a flat limit proportional to the inverse lifetime of a corresponding Minkowski unstable particle as it is usually computed by means of the Fermi golden rule. However we have not yet fully solved the problem of finding a unique Anti-de Sitter normalization to get the right dependence of such lifetime on the speed of the Minkowski particle. The point is that the lifetime is obtained in the Minkowski case as the ratio of two divergent quantities while the Anti-de Sitter amplitude is already finite and it is not completely clear what the "amplitude per unit time" should be in the Anti-de Sitter spacetime. It is an interpretation problem that we leave for further investigation.

Section 2 recalls some well-known facts and fixes some notations. Sections 3, 4, and 5.2 give a precise statement and preliminary discussions of the main mathematical problem to be solved in this paper, and Section 6 gives its solution. Section 7 applies this result to expansion theorems for second kind Gegenbauer functions and the Källén-Lehmann expansion of the product of two free-field two-point functions in AdS (or its covering). Section 8 gives the applications to quantum field theory in AdS mentioned above.

\section{Preliminaries}

The $d$-dimensional real and complex Anti-de Sitter (AdS) space-times with radius $R>0$ are respectively defined as

$$
X_{d}=\left\{x \in \mathbf{R}^{d+1}: x \cdot x=R^{2}\right\} \quad \text { and } \quad X_{d}^{(c)}=\left\{x \in \mathbf{C}^{d+1}: x \cdot x=R^{2}\right\}
$$

where the scalar product $x \cdot y$ is defined as

$$
x \cdot y=x^{0} y^{0}+x^{d} y^{d}-x^{1} x^{1}-\ldots-x^{d-1} x^{d-1}=x^{0} y^{0}+x^{d} y^{d}-\vec{x} \cdot \vec{y} .
$$

The vector $e_{\mu} \in \mathbf{R}^{d+1}$ has coordinates $e_{\mu}^{\nu}=\delta_{\mu \nu} . G_{0}$ (resp. $G_{0}^{(c)}$ ) is the connected component of the unit in the group of real (resp. complex) linear transformations of $\mathbf{R}^{d+1}$ (resp. $\mathbf{C}^{d+1}$ ) which preserve the scalar product (2.2). The future and past tuboids $\mathcal{T}_{1 \pm}$ are given by

$$
\mathcal{T}_{1+}=\left(\mathcal{T}_{1-}\right)^{*}=\left\{x+i y \in X_{d}^{(c)}: y \cdot y>0, \quad y^{0} x^{d}-y^{d} x^{0}>0\right\}
$$

These tuboids are invariant under $G_{0}$. Their properties are studied in detail in [6]. The universal covering spaces of $X_{d}, G_{0}, \mathcal{T}_{1 \pm}$ are respectively denoted $\widetilde{X}_{d}, \widetilde{G}_{0}, \widetilde{\mathcal{T}}_{1 \pm}$. We will assume $d \geq 2$. In this paper, we will take $R=1$ except when it is explicitly stated otherwise.

We denote:

$$
\mathbf{C}_{+}=-\mathbf{C}_{-}=\{z \in \mathbf{C}: \operatorname{Im} z>0\}, \quad \Delta_{1}=\mathbf{C} \backslash[-1,1], \quad \widehat{\Delta}_{1}=\Delta_{1} \cup\{\infty\},
$$

(i.e. $\widehat{\Delta}_{1}$ is the complement of the segment $[-1,1]$ in the Riemann sphere), and $\widetilde{\Delta}_{1}$ the universal cover of $\Delta_{1}$. The image of $\mathcal{T}_{1-} \times \mathcal{T}_{1}$ given by the map $\left(z_{1}, z_{2}\right) \mapsto z_{1} \cdot z_{2}$ is $\Delta_{1}$.

A function $f$ holomorphic in $\mathbf{C}_{+} \cup \mathbf{C}_{-}$is said to have tempered behavior if there are positive integers $M, P$ such that

$$
\mathbf{I} f \mathbf{I}_{M, P} \stackrel{\text { def }}{=} \sup _{z}(1+|z|)^{-M}\left(1+|\operatorname{Im} z|^{-1}\right)^{-P}|f(z)|<\infty \text {. }
$$

Such a function has boundary values $f_{+}$and $f_{-}$on the real axis in the sense of tempered distributions from $\mathbf{C}_{+}$and $\mathbf{C}_{-}$respectively, and we denote $\operatorname{disc} f=f_{+}-f_{-}$. If $T$ is a tempered distribution on $\mathbf{R}$ 
with sufficient decrease at infinity (in particular if it has compact support) then

$$
f(z)=\frac{1}{2 \pi i} \int_{\mathbf{R}} \frac{T(t) d t}{t-z}
$$

is holomorphic with tempered behavior in $\mathbf{C}_{+} \cup \mathbf{C}_{-}$and $\operatorname{disc} f=T$. A sequence $f_{n}$ of functions holomorphic in $\mathbf{C}_{+} \cup \mathbf{C}_{-}$tends to 0 in the sense of functions with tempered behavior if there are positive integers $M, P$ such that $\left|f_{n}\right|_{M, P} \rightarrow 0$. In this case $f_{n \pm} \rightarrow 0$ in the sense of tempered distributions. A function $f$ holomorphic in $\mathcal{T}_{1 \pm}$ is said to have tempered behavior if there are positive integers $M, P$ such that

$$
\mathbf{I} f \mathbf{|}_{M, P} \stackrel{\text { def }}{=} \sup _{z=x+i y \in \mathcal{T}_{1 \pm}}(1+|z|)^{-M}\left(1+|y \cdot y|^{-1}\right)^{-P}|f(z)|<\infty .
$$

If $\phi$ is a neutral scalar local quantum field on $X_{d}$ satisfying standard assumptions (see [6, 7]), there is a function $W$ holomorphic in $\mathcal{T}_{1-} \times \mathcal{T}_{1+}$, and a function $w$ holomorphic with tempered behavior in $\Delta_{1}$, such that, in the sense of tempered distributions, the two-point vacuum expectation value of $\phi$ satisfies

$$
\begin{aligned}
& \mathcal{W}\left(x_{1}, x_{2}\right) \stackrel{\text { def }}{=}\left(\Omega, \phi\left(x_{1}\right) \phi\left(x_{2}\right) \Omega\right)=\lim _{\substack{z_{1} \in \mathcal{T}_{1-}, z_{2} \in \mathcal{T}_{1+} \\
z_{1} \rightarrow x_{1}, \quad z_{2} \rightarrow x_{2}}} W\left(z_{1}, z_{2}\right), \\
& W\left(z_{1}, z_{2}\right)=w\left(z_{1} \cdot z_{2}\right) .
\end{aligned}
$$

Conversely, if $w$ is a function holomorphic with tempered behavior in $\Delta_{1}$, there exists a generalized free field $\phi$ such that (2.8, 2.9) hold (it will satisfy the positivity condition if and only if $\left(z_{1}, z_{2}\right) \mapsto w\left(z_{1} \cdot z_{2}\right)$ is of positive type). In the case of $\widetilde{X}_{d}, w$ is replaced by a function holomorphic on $\widetilde{\Delta}_{1}$; we will mostly consider its restriction to the cut-plane $\mathbf{C} \backslash(-\infty, 1]$.

In the special case of the standard scalar neutral Klein-Gordon field with mass $m$ on $X_{d}$, each of the functions $\mathcal{W}, W$, and $w$ is labelled by a parameter $\nu$ of the form $\nu=n+(d-1) / 2$, where $n$ is an integer $n>(1-d)$, related to the mass by

$$
m^{2}=n(n+d-1)
$$

The function $w_{n+\frac{d-1}{2}}$ is given by

$$
\begin{aligned}
w_{n+\frac{d-1}{2}}(z) & =\frac{e^{-i \pi \frac{d-2}{2}}}{(2 \pi)^{\frac{d}{2}}}\left(z^{2}-1\right)^{-\frac{d-2}{4}} Q_{n+\frac{d-2}{2}}^{\frac{d-2}{2}}(z) \\
& =\frac{\Gamma\left(\frac{d-1}{2}\right)}{2 \pi^{\frac{d+1}{2}}} D_{n}^{\frac{d-1}{2}}(z)
\end{aligned}
$$

Here and in the sequel $z^{\alpha}=\exp (\alpha \log z)$ is defined as holomorphic in $\mathbf{C} \backslash \mathbf{R}_{-}$and $\left(z^{2}-1\right)^{\alpha}$ as $z^{2 \alpha}(1-$ $\left.z^{-2}\right)^{\alpha}$. The function $z \mapsto\left(1-z^{-2}\right)^{\alpha}$ is holomorphic in $\widehat{\Delta}_{1}$. The function $Q_{\alpha}^{\beta}$ is the Legendre function of the second kind (see [15, pp. $122 \mathrm{ff}]$ ) which is defined for complex values of $\alpha$ and $\beta$, and the function $D_{n}^{\lambda}$, a Gegenbauer function of the second kind, is also defined for complex values of $n$ and $\lambda$. The following formulae will play an important role in this paper:

$$
\begin{aligned}
D_{n}^{\lambda}(z) & =\frac{\pi \Gamma(n+2 \lambda)}{\Gamma(\lambda) \Gamma(n+\lambda+1)}(2 z)^{-n-2 \lambda} F\left(\frac{n+2 \lambda}{2}, \frac{n+2 \lambda+1}{2} ; n+\lambda+1 ; \frac{1}{z^{2}}\right) \\
& =\frac{\pi \Gamma(n+2 \lambda)}{\Gamma(\lambda) \Gamma(n+\lambda+1)}(\zeta)^{-n-2 \lambda} F\left(n+2 \lambda, \lambda ; n+\lambda+1 ; \frac{1}{\zeta^{2}}\right),
\end{aligned}
$$

where the variables $z$ and $\zeta$ are related as follows

$$
\zeta=z+\left(z^{2}-1\right)^{\frac{1}{2}}, \quad \zeta^{-1}=z-\left(z^{2}-1\right)^{\frac{1}{2}}, \quad z=\frac{\zeta+\zeta^{-1}}{2}
$$


The above formulae do not require any of the parameters to be an integer, but we will always assume $\operatorname{Re}(n+2 \lambda)>0$ when using them. The equality of (2.13) and (2.14) is explained in Appendix C. The functions $D_{n}^{\lambda}$ are further discussed in Appendix A. Formulae (2.11, 2.12) extend, mutatis mutandis, to the covering $\widetilde{X}_{d}$ of the Anti-de Sitter spacetime, but then $n$ is not any longer required to be an integer.

We denote $E(L)$, with $L>1$, the ellipse with foci \pm 1 given by

$$
E(L)=\left\{\frac{1}{2}\left(\zeta+\zeta^{-1}\right): \zeta \in \mathbf{C},|\zeta|=L\right\} .
$$

The outside $E_{+}(L)$ and inside $E_{-}(L)$ of $E(L)$ are defined by

$$
\begin{aligned}
& E_{+}(L)=\left\{\frac{1}{2}\left(\zeta+\zeta^{-1}\right): \zeta \in \mathbf{C}, \quad|\zeta|>L\right\}, \\
& E_{-}(L)=\left\{\frac{1}{2}\left(\zeta+\zeta^{-1}\right): \quad \zeta \in \mathbf{C}, \quad 1<|\zeta|<L\right\} \cup[-1,1] .
\end{aligned}
$$

We also define $E_{+}(1)=\Delta_{1}$. Note that if $z$ and $\zeta$ are related by 2.15 then $z^{-2}$ is expressible as a series in powers of $\zeta^{-2}$ which converges for $|\zeta|>1$ and vice-versa.

We will frequently use the classical notation $(t)_{k}=t(t+1) \ldots(t+k-1)=\Gamma(t+k) / \Gamma(t)$ if $k$ is an integer $\geq 1,(t)_{k}=1$ if $k \leq 0$.

We will also use the notation

$$
\alpha_{\lambda}(s)=\frac{\Gamma(s+\lambda)}{\Gamma(\lambda) \Gamma(s+1)}=\frac{1}{s \mathrm{~B}(\lambda, s)}
$$

\section{The expansion problem}

If $\mathcal{W}_{m}\left(x_{1}, x_{2}\right)$ denotes the two-point vacuum expectation value of a free neutral scalar Klein-Gordon quantum field with mass $m$ on Minkowski space-time, and if $F\left(x_{1}, x_{2}\right)$ is any function with the same general linear properties as the two-point function of a local field, there exists a tempered weight $\rho$ with support in the positive real axis such that

$$
F\left(x_{1}, x_{2}\right)=\int_{\mathbf{R}_{+}} \rho\left(m^{2}\right) \mathcal{W}_{m}\left(x_{1}, x_{2}\right) d m^{2} .
$$

$\rho$ is called the Källén-Lehmann weight associated to $F$, and it is a positive measure if and only if $F$ is of positive type (see e.g. [14, p. 336]). In particular for any two given masses $m_{1}$ and $m_{2}$

$$
\mathcal{W}_{m_{1}}\left(x_{1}, x_{2}\right) \mathcal{W}_{m_{2}}\left(x_{1}, x_{2}\right)=\int_{\left(m_{1}+m_{2}\right)^{2}}^{\infty} \rho_{\operatorname{Min}}\left(a^{2} ; m_{1}, m_{2}\right) \mathcal{W}_{a}\left(x_{1}, x_{2}\right) d a^{2}
$$

where $\rho_{\text {Min }}\left(a^{2} ; m_{1}, m_{2}\right)$ is easily explicitly computable simply by Fourier transform.

A similar explicit result has been recently obtained by the authors for the de Sitter space-time 3 . The derivation is considerably more involved. This type of formula is of interest in itself from the point of view of special-function theory and also of group theory. In quantum field theory it allows the computation of the lifetime of a de Sitterian unstable particle at first order in perturbation theory: this was carried out in [5, 4, 3].

Can the analogue of (3.2) be explicitly obtained in the case of the AdS space-time? The general problem of constructing the Källén-Lehmann representation for two-point functions of Anti-de Sitterian scalar fields was solved in [8] and a method of calculating the weight outlined there. Having such a representation is of course of importance for calculations in interacting Anti-de Sitter quantum field theories [9, 10].

However to concretely derive an explicit expression for the weights in the quadratic case we study in this paper much additional effort is required. With the notations of Sect. 2, in this paper we intend to establish that

$$
W_{m+\frac{d-1}{2}}\left(z_{1}, z_{2}\right) W_{n+\frac{d-1}{2}}\left(z_{1}, z_{2}\right)=\sum_{l} \rho(l ; m, n) W_{l+\frac{d-1}{2}}\left(z_{1}, z_{2}\right),
$$


with an explicit determination of $\rho(l ; m, n)$. Here $m, n$ and $l$ take integer values. This will be done in the following sections, as well as an extension to the case of the universal cover of the AdS space-time. Equations (3.3) and (2.12) lead to conjecture the following identity

$$
D_{m}^{\lambda}(z) D_{n}^{\lambda}(z)=\sum_{l} c_{\lambda}(m, n \mid l) D_{l}^{\lambda}(z)
$$

in which we only suppose at first that $\operatorname{Re}(m+2 \lambda)>0$ and $\operatorname{Re}(n+2 \lambda)>0$.

Formula (2.13) shows that $z^{n+2 \lambda} D_{n}^{\lambda}(z)$ is holomorphic and even in a neighborhood of $\infty$. It follows that in the rhs of (3.4),$l$ must take values of the form $l=m+n+2 \lambda+2 k$, with integer $k \geq 0$. Inserting Eq. (2.13) into Eq.(3.4) leads to yet another form of the conjectured identity:

$$
\begin{aligned}
& F\left(\frac{m+2 \lambda}{2}, \frac{m+2 \lambda+1}{2} ; m+\lambda+1 ; u\right) F\left(\frac{n+2 \lambda}{2}, \frac{n+2 \lambda+1}{2} ; n+\lambda+1 ; u\right)= \\
= & \sum_{k=0}^{\infty} b_{\lambda}(m, n \mid k) u^{k} F\left(\frac{m+n+4 \lambda+2 k}{2}, \frac{m+n+4 \lambda+2 k+1}{2} ; m+n+3 \lambda+1+2 k ; u\right) .
\end{aligned}
$$

Here we have set $z^{-2}=u$ and adopted the definition

$$
b_{\lambda}(m, n \mid k)=\frac{c_{\lambda}(m, n \mid m+n+2 \lambda+2 k) \alpha_{\lambda}(m+n+3 \lambda+2 k)}{4^{k} \pi \alpha_{\lambda}(m+\lambda) \alpha_{\lambda}(n+\lambda)} .
$$

Using (2.14) instead of (2.13), or more directly the identity (C.5) of Appendix C, we obtain the equivalent (conjectured) identity

$$
\begin{aligned}
& F(m+2 \lambda, \lambda ; m+\lambda+1 ; v) F(n+2 \lambda, \lambda ; n+\lambda+1 ; v)= \\
& =\sum_{k=0}^{\infty} b_{\lambda}(m, n \mid k)(4 v)^{k} F(m+n+4 \lambda+2 k, \lambda ; m+n+3 \lambda+1+2 k ; v) .
\end{aligned}
$$

If $u$ and $v$ are taken to be related by

$$
u=z^{-2}, \quad z=\frac{1}{2}\left(\zeta+\zeta^{-1}\right), \quad v=\zeta^{-2},
$$

the series on the rhs of (3.5) and (3.7) are the same, and the lhs are also the same.

For fixed $m, n$ and $\lambda$, identifying the power series in $u$ which appear on both sides of (3.5) allows an inductive determination of the coefficients $b_{\lambda}(m, n \mid k)$. Identifying the power series in $v$ which appear on both sides of (3.7) leads to an equivalent algebraic problem. This algebraic side of the problem will be discussed in the next section.

\section{The algebraic problem}

It is useful to adopt as independent variables $x=m+2 \lambda, y=n+2 \lambda, \eta=1-\lambda$ instead of $m, n$, and $\lambda$, and to define

$$
f_{k}(x, y, \eta)=4^{k} b_{\lambda}(m, n \mid k)
$$

Eq. (3.5) then becomes

$$
\begin{array}{r}
\sum_{k=0}^{\infty} 4^{-k} f_{k}(x, y, \eta) u^{k} F\left(\frac{x+y+2 k}{2}, \frac{x+y+2 k+1}{2} ; x+y+\eta+2 k ; u\right)= \\
=F\left(\frac{x}{2}, \frac{x+1}{2} ; x+\eta ; u\right) F\left(\frac{y}{2}, \frac{y+1}{2} ; y+\eta ; u\right) .
\end{array}
$$


By using the Legendre duplication formula ([15, 1.3 (15) p. 5]) the relevant hypergeometric series simplifies as follows

$$
F\left(a, a+\frac{1}{2} ; c ; u\right)=\sum_{p=0}^{\infty} \frac{1}{p !} \frac{(2 a)_{2 p}}{(c)_{p}}\left(\frac{u}{4}\right)^{p} .
$$

and equating the coefficients of $u^{r}$ on both sides of Eq. (4.2) gives

$$
\sum_{k=0}^{r} f_{k}(x, y, \eta) \frac{(x+y+2 k)_{2(r-k)}}{(x+y+\eta+2 k)_{r-k}(r-k) !}=\sum_{p=0}^{r} \frac{(x)_{2 p}(y)_{2 r-2 p}}{(x+\eta)_{p} p !(y+\eta)_{r-p}(r-p) !} .
$$

Note the convolution structure of the rhs; the coefficients there can be seen to be a one-parameter deformation of the binomial coefficient $\left(\begin{array}{c}x+2 p-1 \\ p\end{array}\right)$.

$$
\left(\begin{array}{c}
x+2 p-1 \\
p
\end{array}\right)_{\eta}=\frac{(x)_{2 p}}{(x+\eta)_{p} p !}=\frac{\Gamma(x+2 p)}{\Gamma(x+\eta+p) \Gamma(p+1)} \frac{\Gamma(x+\eta)}{\Gamma(x)} .
$$

The system (4.4) is suitable for an iterative solution of the problem.

Setting $r=0$ gives $f_{0}(x, y, \eta)=1$. For any $r>0$, the coefficient of $f_{r}(x, y, \eta)$ in Eq. (4.4) is 1 so that (4.4) provides an expression of $f_{r}(x, y, \eta)$ in terms of all the $f_{k}(x, y, \eta)$ with $k<r$.

It is clear by induction that all $f_{r}(x, y, \eta)$ are rational functions of the variables $x, y$, and $\eta$, the degrees of numerator and denominator depending on $r$. An equivalent form of the system of equations (4.4) is obtained by defining

$$
a_{r}(x, y, \eta)=r ! f_{r}(x, y, \eta)(x+\eta)_{r}(y+\eta)_{r}(x+y+\eta)_{2 r} .
$$

Then (4.4) gives

$$
\begin{aligned}
& \sum_{k=0}^{r} a_{k}(x, y, \eta)\left(\begin{array}{l}
r \\
k
\end{array}\right)(x+y+2 k)_{2(r-k)}(x+\eta+k)_{r-k}(y+\eta+k)_{r-k}(x+y+\eta+k+r)_{r-k}= \\
& =(x+y+\eta)_{2 r} \sum_{p=0}^{r}\left(\begin{array}{l}
r \\
p
\end{array}\right)(x)_{2 p}(x+\eta+p)_{r-p}(y)_{2 r-2 p}(y+\eta+r-p)_{p} .
\end{aligned}
$$

The case $r=0$ gives $a_{0}(x, y, \eta)=1$. Since the binomial coefficient is an integer, for $r>0$ the function $a_{r}(x, y, \eta)$ is seen by induction to be a polynomial with integer coefficients in the variables $x, y$, and $\eta$, with degree $\leq 5 r$, degree in $x$ or $y \leq 4 r$, and degree in $\eta \leq 3 r$.

With the same change of variables Eq. (3.7) becomes

$$
\begin{aligned}
& F(x, 1-\eta ; x+\eta ; v) F(y, 1-\eta ; y+\eta ; v)= \\
& =\sum_{k=0}^{\infty} f_{k}(x, y, \eta) v^{k} F(x+y+2 k, 1-\eta ; x+y+\eta+2 k ; v) .
\end{aligned}
$$

Equating the terms in $v^{r}$ on both sides of (4.8) shows that for every integer $r \geq 0$,

$$
\sum_{k=0}^{r} f_{k}(x, y, \eta) \frac{(x+y+2 k)_{r-k}(1-\eta)_{r-k}}{(x+y+\eta+2 k)_{r-k}(r-k) !}=\sum_{p, q \geq 0, p+q=r} \frac{(x)_{p}(1-\eta)_{p}(y)_{q}(1-\eta)_{q}}{(x+\eta)_{p}(y+\eta)_{q} p ! q !}
$$

and, with the same definition of $a_{k}(x, y, \eta)$ as in (4.6),

$$
\begin{aligned}
& \sum_{k=0}^{r}\left(\begin{array}{l}
r \\
k
\end{array}\right) a_{k}(x, y, \eta) \times \\
& \times(x+\eta+k)_{r-k}(y+\eta+k)_{r-k}(x+y+\eta+r+k)_{r-k}(x+y+2 k)_{r-k}(1-\eta)_{r-k}= \\
& =(x+y+\eta)_{2 r} \sum_{p, q \geq 0, p+q=r}\left(\begin{array}{l}
r \\
p
\end{array}\right)(x)_{p}(1-\eta)_{p}(x+\eta+p)_{q}(y)_{q}(1-\eta)_{q}(y+\eta+q)_{p} .
\end{aligned}
$$


Of course the systems (4.4), (4.7), (4.9) and (4.10) are all equivalent. Note that from the form (4.10) it can be seen by induction that $a_{r}(x, y, \eta)$ is of degree $\leq 3 r$ in $x$ and of degree $\leq 3 r$ in $y$.

From now on, $f_{r}(x, y, \eta)$ and $a_{r}(x, y, \eta)$ will denote the solutions of the systems (4.4) or (4.9) and (4.7) or (4.10), respectively (they are, of course, related by (4.6)), and $c_{\lambda}(m, n \mid l)$ will denote the quantity obtained from this solution by retracing through Eqs. (4.1) and (3.6).

The following theorem will be proved:

Theorem 4.1 The solution of the system (4.4) or (4.9) is explicitly given by

$$
f_{k}(x, y, \eta)=\widetilde{f}_{k}(x, y, \eta) \stackrel{\text { def }}{=} \frac{(x)_{k}(y)_{k}(x+y+2 \eta-1+k)_{k}(1-\eta)_{k}}{(x+\eta)_{k}(y+\eta)_{k}(x+y+\eta-1+k)_{k} k !} .
$$

Equivalently, the solution of (4.7) or $(4.10)$ is explicitly given by $a_{k}(x, y, \eta)=\widetilde{a}_{k}(x, y, \eta)$, with $\widetilde{a}_{0}(x, y, \eta)=1$ and, for $k \geq 1$,

$$
\widetilde{a}_{k}(x, y, \eta) \stackrel{\text { def }}{=}(x+y+\eta+2 k-1)(x)_{k}(y)_{k}(x+y+\eta)_{k-1}(x+y+2 \eta-1+k)_{k}(1-\eta)_{k}
$$

Finally redefining $c_{\lambda}(m, n \mid l)$ by inverting Eqs. (4.1) and (3.6), we get, for $l=m+n+2 \lambda+2 k, k a$ non-negative integer,

$$
c_{\lambda}(m, n \mid l)=\frac{\pi \alpha_{\lambda}\left(\frac{l+m-n}{2}\right) \alpha_{\lambda}\left(\frac{l-m+n}{2}\right) \alpha_{\lambda}\left(\frac{l+m+n}{2}+\lambda\right) \alpha_{\lambda}\left(\frac{l-m-n}{2}-\lambda\right)}{\alpha_{\lambda}(l) \alpha_{\lambda}(l+\lambda)}
$$

In this statement, (4.12) is an identity between polynomials in $x, y$ and $\eta$, but (4.11) must be understood as an identity between rational functions, and (4.13) as an identity between meromorphic functions.

It is remarkable that the polynomial $\widetilde{a}_{k}(x, y, \eta)$ is completely factorized into a product of polynomials of the first degree (with integer coefficients) in all variables. This gives the identities (4.7) and (4.10) the appearance of a small algebraic miracle.

We do not have, at the moment, a purely algebraic proof of this theorem. Instead it will be proved by a roundabout analytic method. We will use the following remark:

Remark 4.1 (Algebraic continuation) Let $S\left(x_{1}, \ldots, x_{N}\right)$ be a complex polynomial in $N$ variables of degree $d_{j}$ in $x_{j}$ for all $j$. Suppose that $S$ vanishes on $\mathcal{A}_{1} \times \ldots \times \mathcal{A}_{N}$ where, for each $j, \mathcal{A}_{j} \subset \mathbf{C}$ has more than $d_{j}$ distinct elements. Then, as it is easy to see by induction on $N, S$ is identically 0 . This is in particular true if all the $\mathcal{A}_{j}$ are infinite.

Let us now assume that, for some fixed $k>0$, the statement of the theorem, in the form (4.13), has been proved under the following assumptions: $m, n, r=\frac{1}{2}-\lambda$ are non-negative integers and

$$
m-2 r \geq 0, \quad n-2 r \geq 0, \quad r \geq 1
$$

(It follows that $l=m+n+2 \lambda+2 k$ is an integer verifying $l-m>0, l-n>0, l-2 r>0$.) This is equivalent to having proved (4.11) and (4.12) (for this value of $k$ ) under the conditions that $x, y, \eta-\frac{1}{2}$ are integers satisfying

$$
\eta-\frac{1}{2} \geq 1, \quad x-1 \geq 0, \quad y-1 \geq 0 .
$$

Then, by applying Remark 4.1 to $a_{k}(x, y, \eta)-\widetilde{a}_{k}(x, y, \eta)$, considered as a polynomial in $x$, $y$, $\eta$, we conclude that this polynomial is identically 0 , i.e. that, for that value of $k$, the theorem holds for all values of $x, y, \eta$. This will be done, and Theorem 4.1 will be proved, in Sect. 6 . 


\section{Checking the conjecture}

\subsection{Computer proofs at fixed $r$}

According to (4.7) proving Theorem 4.1 is equivalent to proving that, for every integer $r>0$, the two polynomials with integer coefficients

$$
\begin{gathered}
L_{r}(x, y, \eta)=\sum_{k=0}^{r} \frac{r !}{k !(r-k) !} \widetilde{a}_{k}(x, y, \eta)(x+y+2 k)_{2(r-k)}(x+\eta+k)_{r-k}(y+\eta+k)_{r-k} \times \\
\times(x+y+\eta+k+r)_{r-k},
\end{gathered}
$$

and

$$
R_{r}(x, y, \eta)=(x+y+\eta)_{2 r} \sum_{p, q \geq 0, p+q=r} \frac{r !}{p ! q !}(x)_{2 p}(x+\eta+p)_{q}(y)_{2 q}(y+\eta+q)_{p}
$$

coincide. Here $\widetilde{a}_{0}(x, y, \eta)=1$, and, for $k \geq 1, \widetilde{a}_{k}(x, y, \eta)$ is given by (4.12). Equivalently, by (4.10),

$$
\begin{gathered}
L_{r}^{\prime}(x, y, \eta)=\sum_{k=0}^{r} \frac{r !}{k !(r-k) !} \widetilde{a}_{k}(x, y, \eta)(x+\eta+k)_{r-k}(y+\eta+k)_{r-k}(1-\eta)_{r-k} \times \\
\times(x+y+\eta+k+r)_{r-k}(x+y+2 k)_{r-k}
\end{gathered}
$$

and

$$
R_{r}^{\prime}(x, y, \eta)=(x+y+\eta)_{2 r} \sum_{p, q \geq 0, p+q=r} \frac{r !}{p ! q !}(x)_{p}(1-\eta)_{p}(x+\eta+p)_{q}(y)_{q}(1-\eta)_{q}(y+\eta+q)_{p}
$$

must coincide. Let $r \geq 1$ be fixed. $L_{r}$ and $R_{r}$ have degree $\leq 4 r$ in $x$, degree $\leq 4 r$ in $y$, and degree $\leq 3 r$ in $\eta$. By Remark 4.1 it suffices to check that $L_{r}(x, y, \eta)$ and $R_{r}(x, y, \eta)$ take the same values when $(x, y, \eta)$ runs over a set of the form $\mathcal{A}_{1} \times \mathcal{A}_{2} \times \mathcal{A}_{3}$, where the $\mathcal{A}_{j}$ are finite sets with (at least) $4 r+1,4 r+1$ and $3 r+1$ elements, respectively. For example we can take $\mathcal{A}_{1}=\mathcal{A}_{2}=\mathbf{Z} \cap[-4 r, 0]$, $\mathcal{A}_{3}=\mathbf{Z} \cap[0,3 r]$. It is therefore possible to write a program to prove the conjecture for any fixed $r$ using some form of arbitrarily large integer, such as GNU's GMP's mpz_t or Java's BigInteger. A few easy remarks allow to omit checking for some of these values: the symmetry in $x$ and $y$, the identities

$$
L_{r}(0, y, \eta)=R_{r}(0, y, \eta)=(y)_{2 r}(y+\eta)_{2 r}(\eta)_{r}, \quad L_{r}(x, 0, \eta)=R_{r}(x, 0, \eta)=(x)_{2 r}(x+\eta)_{2 r}(\eta)_{r}
$$

and the fact that $L_{r}(x, y, \eta)=R_{r}(x, y, \eta)=0$ if $x$ and $y$ are negative integers such that $2 r+x+y>0$. It is thus sufficient to check only the integer values of $x, y$ and $\eta$ such that

$$
-4 r \leq x \leq-r, \quad x \leq y<0, \quad y \leq-x-2 r, \quad 0 \leq \eta \leq 3 r .
$$

Similar remarks apply to the second form of the problem, $L_{r}^{\prime}(x, y, \eta)=R_{r}^{\prime}(x, y, \eta)$, which is even more favorable as we can take $\mathcal{A}_{1}=\mathcal{A}_{2}=\mathbf{Z} \cap[-3 r, 0], \mathcal{A}_{3}=\mathbf{Z} \cap[0,4 r]$, and use the easily checked identity

$$
L_{r}^{\prime}(x, y, 0)=R_{r}^{\prime}(x, y, 0)=(r+1) !(x)_{r}(y)_{r}(x+y)_{2 r} .
$$

Note also that if $\eta$ is an integer and $0 \leq \eta-1<r / 2$, then $L_{r}^{\prime}(x, y, \eta)=R_{r}^{\prime}(x, y, \eta)=0$. It is therefore sufficient to check only the integer values of $x, y$ and $\eta$ such that

$$
-3 r \leq x \leq-\frac{r}{2}, \quad x \leq y<0, \quad y \leq-x-r, \quad 1+\frac{r}{2} \leq \eta \leq 4 r
$$

We have used such programs to prove the theorem for $r=1, \ldots, 51, r=101,151,171,201,250,3011$. Of course these proofs for selected values of $r$ do not constitute a computer-proof of Theorem 4.1, but they at least serve as a check on the calculations involved in the actual proof (Sect. 6).

\footnotetext{
${ }^{1}$ Computing time appears to increase very roughly like $r^{4}$. On a machine with two INTEL XEON E5504 processors at $2 \mathrm{GH}$, each with 4 cores, $r=301$ (first form) took approximately 55 days.
} 


\subsection{The three dimensional case $d=3$}

The three-dimensional case $\lambda=\frac{d-1}{2}=1$ (i.e. $\eta=0$ ) allows for a simple verification of the different forms of the conjecture.

Let us verify at first formula (3.4). Here the conjectured coefficients do not depend on $n, m$ and $l$ and have all the same value

$$
c_{1}(m, n \mid l)=\pi
$$

The three-dimensional Gegenbauer function (2.13) is most simply written by using the variable $z=$ ch $t$ :

$$
D_{n}^{1}(\operatorname{ch} t)=\frac{\pi}{2} \frac{e^{-(n+1) t}}{\operatorname{sh} t}
$$

Suppose that $\operatorname{Re} t>0$; it follows that

$$
\begin{aligned}
D_{m}^{1}(\operatorname{ch} t) D_{n}^{1}(\operatorname{ch} t) & =\frac{\pi^{2}}{4} \frac{e^{-(m+n+2) t}}{\operatorname{sh}^{2} t}=\frac{\pi^{2}}{2} \frac{e^{-t}}{1-e^{-2 t}} \frac{e^{-(m+n+2) t}}{\operatorname{sh} t}= \\
& =\frac{\pi^{2}}{2} \sum_{k=0}^{\infty} \frac{e^{-(m+n+3+2 k) t}}{\operatorname{sh} t}=\pi \sum_{k=0}^{\infty} D_{m+n+2+2 k}^{1}(\operatorname{ch} t)
\end{aligned}
$$

and the conjectured formula (3.4) is readily verified.

It is equally simple to verify directly the conjecture in the form given by Eq. (3.5); the conjectured coefficients are $b_{1}(m, n \mid k)=4^{-k}$. Since

$$
F\left(a, a+\frac{1}{2} ; 2 a ; u\right)=\frac{1}{\sqrt{1-u}}\left(\frac{2}{1+\sqrt{1-u}}\right)^{2 a-1}
$$

it follows that

$$
\begin{aligned}
& \sum_{k}\left(\frac{u}{4}\right)^{k} F\left(\frac{m+n+4+2 k}{2}, \frac{m+n+5+2 k}{2} ; m+n+4+2 k ; u\right)= \\
& =\frac{1}{\sqrt{1-u}}\left(\frac{2}{1+\sqrt{1-u}}\right)^{m+n+3} \sum_{k}\left(\frac{\sqrt{u}}{1+\sqrt{1-u}}\right)^{2 k}=\frac{1}{1-u}\left(\frac{2}{1+\sqrt{1-u}}\right)^{m+n+2}= \\
& =F\left(\frac{m+2}{2}, \frac{m+3}{2} ; m+2 ; u\right) F\left(\frac{n+2}{2}, \frac{n+3}{2} ; n+2 ; u\right)
\end{aligned}
$$

The second form of the algebraic problem is also immediately verified by Eq. (5.7). Verifying the conjecture order by order as in Eq. (4.4) is a little trickier already in this elementary case. At order $r$ the validity of the conjecture amounts in $d=3$ to the following identity:

$$
\sum_{s=0}^{r}\left(\begin{array}{c}
x+y+2 r-1 \\
r-s
\end{array}\right)=\sum_{p=0}^{r}\left(\begin{array}{c}
x+2 p-1 \\
p
\end{array}\right)\left(\begin{array}{c}
y+2 r-2 p-1 \\
r-p
\end{array}\right) .
$$

A formula closely similar to this one has been proven in [11, Eq. (5.13-14)]. A direct bijective proo 2 is as follows. The lhs counts random walks of length $x+y+2 r-1$, starting at height 0 and ending at height $\geq x+y-1$. The same set of walks is counted differently at the rhs. First, one performs a last-passage decomposition at height $x-1$; the length is of the form $x-1+2 p$ with $0 \leq p \leq r$. This yields immediately

$$
\sum_{k=0}^{r}\left(\begin{array}{c}
x+y+2 r-1 \\
k
\end{array}\right)=\sum_{p=0}^{r}\left(\begin{array}{c}
x+2 p-1 \\
p
\end{array}\right) B(y, r-p)
$$

\footnotetext{
${ }^{2}$ This proof has been communicated to us by J. Bouttier and E. Guitter. We thank them for discussions on this point
} 
where $B(y, q)$ is the number of positive walks of length $y-1+2 q$ starting at height 0 and ending at height $\geq y-1$. It remains to show that $B(y, q)=\left(\begin{array}{c}y-1+2 q \\ q\end{array}\right)$. Consider such a walk ending at height $y-1+2 j$, and consider the last passages at heights $0,1, \ldots, j-1$ : "flip" the corresponding steps (from up to down). This defines a bijection with walks of length $y-1+2 q$ starting at height 0 and ending at $y-1$ whose minimal height is $-j$ (the flipped steps become the first passages at heights $-1,-2, \cdots,-j$ ). Summing over $j$ the result follows.

\subsection{Remark}

The validity of the relation (5.14) is already guaranteed by the previous Eq. (5.13). Similarly, the proof of the full conjecture will imply the validity of the following one parameter deformation of Eq. (5.14)

$$
\begin{aligned}
& \sum_{k=0}^{r} \frac{(x)_{k}}{(x+\eta)_{k}} \frac{(y)_{k}}{(y+\eta)_{k}} \frac{(x+y+2 \eta-1+k)_{k}(x+y+2 k)_{r-k}}{(x+y+\eta-1+k)_{k}(x+y+\eta+2 k)_{r-k}} \frac{(1-\eta)_{k}(1-\eta)_{r-k}}{k !(r-k) !}\left(\begin{array}{c}
x+y+2 r-1 \\
k
\end{array}\right)_{\eta}= \\
& =\sum_{p=0}^{r}\left(\begin{array}{l}
x \\
p
\end{array}\right)_{\eta}\left(\begin{array}{c}
y \\
r-p
\end{array}\right)_{\eta}
\end{aligned}
$$

At the moment we do not know if any combinatorial interpretation of this generalization of Eq. (5.14) does exist.

\section{An analytic version of the problem}

In this section $\lambda$ will be of the form $\lambda=\frac{1}{2}-r$, and $r, m$, and $n$ will be integers such that

$$
r \geq 1, \quad m-2 r \geq 0, \quad n-2 r \geq 0 .
$$

Under these conditions the function

$$
F(z)=\left(z^{2}-1\right)^{\lambda-\frac{1}{2}} D_{m}^{\lambda}(z) D_{n}^{\lambda}(z)
$$

is holomorphic on $\widehat{\Delta}_{1}$ and satisfies the hypotheses of Theorem A.3. stated in Appendix $\mathrm{A}$ with $N=$ $m+n+2-2 r, N-1=m+n+1-2 r \geq 2 r+1$. The theorem then asserts that

$$
\left(z^{2}-1\right)^{\lambda-\frac{1}{2}} D_{m}^{\lambda}(z) D_{n}^{\lambda}(z)=\left(z^{2}-1\right)^{\lambda-\frac{1}{2}} \sum_{l=m+n+2 \lambda}^{\infty} \widehat{c}_{\lambda}(m, n \mid l) D_{l}^{\lambda}(z)
$$

holds with uniform convergence on every compact subset of $\widehat{\Delta}_{1}$, with

$$
\widehat{c}_{\lambda}(m, n \mid l)=\frac{2^{2 \lambda-1}(l+\lambda) \Gamma(l+1) \Gamma(\lambda)^{2}}{i \pi^{2} \Gamma(l+2 \lambda)} \int_{E(L)} C_{l}^{\lambda}(z)\left(z^{2}-1\right)^{\lambda-\frac{1}{2}} D_{m}^{\lambda}(z) D_{n}^{\lambda}(z) d z .
$$

Here $E(L)$ is the ellipse defined in (2.16) for any $L>1$, but it can, of course, be replaced by any continuous contour homotopic to it in $\Delta_{1}$. The index $l$ takes integer values. It follows from $C_{l}^{\lambda}(z)=$ $(-1)^{l} C_{l}^{\lambda}(-z)$ that $\widehat{c}_{\lambda}(m, n \mid l)$ vanishes unless $l=m+n+2 \lambda+2 k$ with an integer $k \geq 0$. Since the Laurent coefficients of both sides of (6.3) can be obtained by Cauchy integrals on some circle centered at 0 with radius $R>1$, and the expansion is uniform on this circle, the coefficients $\widehat{c}_{\lambda}(m, n \mid l)$ can be obtained by identifying these Laurent series. Hence $\widehat{c}_{\lambda}(m, n \mid l)=c_{\lambda}(m, n \mid l)$, where $c_{\lambda}(m, n \mid l)$ is the solution of the algebraic problem considered in sect. 4, and (6.4) gives a new expression for this solution under the special conditions we have imposed.

Since the function $F$ is holomorphic in $\Delta_{1}$ and has tempered behavior, we find by contour deformation

$$
\int_{E(L)} C_{n}^{\frac{1}{2}-r}(z) F(z) d z=-\int \operatorname{disc}\left[C_{n}^{\frac{1}{2}-r}(x) F(x)\right] d x .
$$


Therefore

$$
\begin{aligned}
c_{\frac{1}{2}-r}(m, n \mid l) & =\frac{-2^{-2 r}\left(l+\frac{1}{2}-r\right) \Gamma(l+1) \Gamma\left(\frac{1}{2}-r\right)^{2}}{i \pi^{2} \Gamma(l+1-2 r)} \int \operatorname{disc}\left[\left(x^{2}-1\right)^{-r} C_{l}^{\frac{1}{2}-r}(x) D_{m}^{\frac{1}{2}-r}(x) D_{n}^{\frac{1}{2}-r}(x)\right] d x \\
& =\frac{-2^{-2 r}\left(l+\frac{1}{2}-r\right) \Gamma(l+1) \Gamma\left(\frac{1}{2}-r\right)^{2}}{i \pi^{2} \Gamma(l+1-2 r)} \int\left(x^{2}-1\right)^{-r} C_{l}^{\frac{1}{2}-r}(x) \operatorname{disc}\left[D_{m}^{\frac{1}{2}-r}(x) D_{n}^{\frac{1}{2}-r}(x)\right] d x .
\end{aligned}
$$

The last equality holds because in the case $r \geq 1, l \geq 2 r$, which we are considering, $\left(x^{2}-1\right)^{-r} C_{l}^{\frac{1}{2}-r}(x)$ is a polynomial. Under our assumptions, $D_{m}^{\frac{1}{2}-r}$ and $D_{n}^{\frac{1}{2}-r}$ have continuous boundary values on the real axis (see Appendix A, subsect. A.3). Thus the integrand of (6.6) or (6.7) is a continuous function on $\mathbf{R}$ with support in $[-1,1]$.

Since the boundary values of $D_{k}^{\frac{1}{2}-r}$ are continuous, it suffices to study disc $\left[D_{m}^{\frac{1}{2}-r}(x) D_{n}^{\frac{1}{2}-r}(x)\right]$ on the open interval $(-1,1)$. It is given by

$$
\operatorname{disc}\left[D_{m}^{\frac{1}{2}-r}(x) D_{n}^{\frac{1}{2}-r}(x)\right]=\left[\operatorname{disc} D_{m}^{\frac{1}{2}-r}(x)\right] \mathbf{D}_{n}^{\frac{1}{2}-r}(x)+\mathbf{D}_{m}^{\frac{1}{2}-r}(x)\left[\operatorname{disc} D_{n}^{\frac{1}{2}-r}(x)\right] .
$$

Here $\mathbf{D}_{k}^{\frac{1}{2}-r}(x)$ is defined as $\frac{1}{2} D_{k}^{\frac{1}{2}-r}(x+i 0)+\frac{1}{2} D_{k}^{\frac{1}{2}-r}(x-i 0)$ and is discussed in Appendix $\mathrm{A}$ as well as $\operatorname{disc} D_{n}^{\frac{1}{2}-r}(x)$. The integrand of (6.7) is equal to

$$
\begin{gathered}
I=I_{m, n}+I_{n, m}, \\
I_{m, n}=\frac{(-i) \pi^{3 / 2}(-2)^{r} \Gamma(n-2 r+1)}{\Gamma(n+1) \Gamma\left(\frac{1}{2}-r\right)} \theta(1+x) \theta(1-x) \times \\
\times\left(1-x^{2}\right)^{-r / 2} C_{l}^{\frac{1}{2}-r}(x) C_{m}^{\frac{1}{2}-r}(x) \mathbf{Q}_{n-r}^{r}(x) .
\end{gathered}
$$

Since $l>2 r, I_{m, n}$ is of the form

$$
I_{m, n}=h(x) \mathbf{Q}_{n-r}^{r}(x), \quad h(x)=\theta(1+x) \theta(1-x)\left(1-x^{2}\right)^{3 r / 2} \varphi(x),
$$

where $\varphi$ is a polynomial. Thus

$$
\begin{aligned}
& \left(1-x^{2}\right)^{r / 2} h(x)=\theta(1+x) \theta(1-x)\left(1-x^{2}\right)^{2 r} \varphi(x) \\
& \left(\frac{d}{d x}\right)^{r}\left[h(x)\left(1-x^{2}\right)^{r / 2}\right]=\theta(1+x) \theta(1-x)\left(\frac{d}{d x}\right)^{r}\left[\left(1-x^{2}\right)^{2 r} \varphi(x)\right] .
\end{aligned}
$$

The last function (6.13) is continuous on $\mathbf{R}$ with support in $[-1,1]$, thus belongs to $L^{2}(\mathbf{R})$. For reasons explained in Appendix A (subsect. A.5), it is legitimate to make the substitution

$$
\mathbf{Q}_{n-r}^{r}(x)=\sum_{k=2 r}^{\infty} a_{r}(k, n) \mathbf{P}_{k-r}^{r}(x)
$$

where

$$
a_{r}(k, n)=a(k-r, n-r),
$$

and

$$
a(k, n)= \begin{cases}\frac{1}{k-n}+\frac{1}{k+n+1} & \text { if } k-n \text { is odd } \\ 0 & \text { if } k-n \text { is even }\end{cases}
$$


(These $a_{r}$ have nothing to do with those used in sect. 4). Using the identity (see [15, 3.15.1 (4), p. 175] and (A.21)

$$
\mathbf{P}_{k-r}^{r}(x)=\frac{2^{-r} \pi^{-1 / 2} \Gamma\left(\frac{1}{2}-r\right) \Gamma(k+1)}{\Gamma(k-2 r+1)}\left(1-x^{2}\right)^{-r / 2} C_{k}^{\frac{1}{2}-r}(x),
$$

we obtain

$$
\begin{gathered}
c_{\frac{1}{2}-r}(m, n \mid l)=R(m, n \mid l)+R(n, m \mid l), \\
R(m, n \mid l)=\frac{(-4)^{-r}\left(l+\frac{1}{2}-r\right) \Gamma(l+1) \Gamma\left(\frac{1}{2}-r\right)^{2}}{\pi \Gamma(l+1-2 r)} \times \\
\sum_{k=2 r}^{\infty} \frac{\Gamma(n-2 r+1) \Gamma(k+1)}{\Gamma(k-2 r+1) \Gamma(n+1)} a_{r}(k, n) H(r ; m, k, l),
\end{gathered}
$$

with

$$
H\left(r ; n_{1}, n_{2}, n_{3}\right)=\int_{-1}^{1}\left(1-x^{2}\right)^{-r} C_{n_{1}}^{\frac{1}{2}-r}(x) C_{n_{2}}^{\frac{1}{2}-r}(x) C_{n_{3}}^{\frac{1}{2}-r}(x) d x .
$$

An explicit expression for $H\left(r ; n_{1}, n_{2}, n_{3}\right)$ has been given by Hsü ([16]) for any triple of integers $n_{1}, n_{2}, n_{3}$ and any complex $r$ with $\operatorname{Re} r<1$. (See also the very interesting discussions in [12] and [13] for the connection with a theorem of Dougall.) In Appendix B this result is shown also to hold for all integer values of $r>0$ such that $n_{j} \geq 2 r$. Recall that for such values, each of the polynomials $C_{n_{j}-r}^{r}(x)$ is divisible by $\left(1-x^{2}\right)^{r}$. It is found that $H\left(r ; n_{1}, n_{2}, n_{3}\right)$ is equal to 0 unless $n_{j} \leq n_{k}+n_{l}$ for any permutation $(j, k, l)$ of $(1,2,3)$, and $2 s=n_{1}+n_{2}+n_{3}$ is an even integer. Otherwise,

$$
\begin{aligned}
& H\left(r ; n_{1}, n_{2}, n_{3}\right)= \\
& 2^{r} \pi \frac{\Gamma(s+1-2 r)}{\Gamma\left(s+\frac{3}{2}-r\right) \Gamma\left(\frac{1}{2}-r\right)} \frac{\Gamma\left(s-n_{1}+\frac{1}{2}-r\right)}{\Gamma\left(s-n_{1}+1\right) \Gamma\left(\frac{1}{2}-r\right)} \frac{\Gamma\left(s-n_{2}+\frac{1}{2}-r\right)}{\Gamma\left(s-n_{2}+1\right) \Gamma\left(\frac{1}{2}-r\right)} \frac{\Gamma\left(s-n_{3}+\frac{1}{2}-r\right)}{\Gamma\left(s-n_{3}+1\right) \Gamma\left(\frac{1}{2}-r\right)}(6.21)
\end{aligned}
$$

An important consequence of this is that the summation in (6.19) extends only over values of $k$ such that $l-m \leq k \leq l+m$. 


\subsection{Evaluation}

We will now proceed to the actual evaluation of $R(m, n \mid l)$. To this end we consider the meromorphic function $x \mapsto s(x)$ defined by

$$
s(x)=\frac{\Gamma\left(\frac{l+m-x+1-2 r}{2}\right)}{\Gamma\left(\frac{l+m-x+2}{2}\right)} \frac{\Gamma\left(\frac{l+m+x+2-4 r}{2}\right)}{\Gamma\left(\frac{l+m+x+3-2 r}{2}\right)} \frac{\Gamma\left(\frac{l-m+x+1-2 r}{2}\right)}{\Gamma\left(\frac{l-m+x+2}{2}\right)} \frac{\Gamma\left(\frac{l-m-x}{2}\right)}{\Gamma\left(\frac{l-m-x+1+2 r}{2}\right)} \frac{\Gamma(x+1)}{\Gamma(x-2 r+1)}
$$

where we continue to suppose $l \geq m+n+2 \lambda, \lambda=\frac{1}{2}-r, m, n$ and $r$ integers, $r \geq 1, m-2 r \geq 0$, $n-2 r \geq 0$, which imply $l-m>0$. $s(x)$ is the ratio of two polynomials,

$$
s(x)=\frac{p(x)}{q(x)},
$$

where

$$
\begin{aligned}
p(x) & =\frac{\Gamma\left(\frac{l+m+1-x-2 r}{2}\right)}{\Gamma\left(\frac{l-m+1-x+2 r}{2}\right)} \frac{\Gamma\left(\frac{l+m+x+2-4 r}{2}\right)}{\Gamma\left(\frac{l-m+x+2}{2}\right)} \frac{\Gamma(x+1)}{\Gamma(x-2 r+1)}= \\
& =\left(\frac{l-m+1-x+2 r}{2}\right)_{m-2 r}\left(\frac{l-m+x+2}{2}\right)_{m-2 r}(x-2 r+1)_{2 r}, \\
q(x) & =\frac{\Gamma\left(\frac{l+m+2-x}{2}\right)}{\Gamma\left(\frac{l-m-x}{2}\right)} \frac{\Gamma\left(\frac{l+m+x+3-2 r}{2}\right)}{\Gamma\left(\frac{l-m+x+1-2 r}{2}\right)}=\left(\frac{l-m-x}{2}\right)_{m+1}\left(\frac{l-m+x+1-2 r}{2}\right)_{m+1} .
\end{aligned}
$$

The degree of $p(x)$ is $2 m-2 r$ while the degree of $q(x)$ is $2 m+2$. The symmetry $s(x)=s(-x+2 r-1)$ implies that $s(x)$ admits a partial fraction decomposition of the following form:

$$
s(x)=\sum_{k=l-m, k \geq 2 r}^{l+m} \sigma(k, l, m)\left(\frac{1}{k-x}+\frac{1}{k+x-2 r+1}\right),
$$

where the sum runs over the zeros of the polynomial $\left(\frac{l-m-x}{2}\right)_{m+1}$ namely

$$
k=l-m, l-m+2, \ldots, l+m
$$

The coefficient $\sigma(k, l, m)$ may be computed by taking the residue

$$
\sigma(k, l, m)=\lim _{x \rightarrow k}(k-x) s(x) .
$$

Since

$$
\Gamma(-k+\epsilon) \sim \frac{(-1)^{k}}{\Gamma(k+1)} \frac{1}{\epsilon}
$$

we find

$$
\begin{aligned}
& \sigma(k, l, m)=\lim _{x \rightarrow k}(k-x) s(x)= \\
& =\frac{2(-1)^{\frac{l-m-k}{2}}}{\Gamma\left(\frac{m+k-l+2}{2}\right)} \frac{\Gamma\left(\frac{l+m-k+1-2 r}{2}\right)}{\Gamma\left(\frac{l+m-k+2}{2}\right)} \frac{\Gamma\left(\frac{l+m+k+2-4 r}{2}\right)}{\Gamma\left(\frac{l+m+k+3-2 r}{2}\right)} \frac{\Gamma\left(\frac{l-m+k+1-2 r}{2}\right)}{\Gamma\left(\frac{l-m+k+2}{2}\right)} \frac{1}{\Gamma\left(\frac{l-m-k+1+2 r}{2}\right)} \frac{\Gamma(k+1)}{\Gamma(k-2 r+1)}= \\
& =\frac{2}{\pi}(-1)^{r} \frac{\Gamma\left(\frac{m+k-l+1-2 r}{2}\right)}{\Gamma\left(\frac{m+k-l+2}{2}\right)} \frac{\Gamma\left(\frac{l+m-k+1-2 r}{2}\right)}{\Gamma\left(\frac{l+m-k+2}{2}\right)} \frac{\Gamma\left(\frac{l+m+k+2-4 r}{2}\right)}{\Gamma\left(\frac{l+m+k+3-2 r}{2}\right)} \frac{\Gamma\left(\frac{l-m+k+1-2 r}{2}\right)}{\Gamma\left(\frac{l-m+k+2}{2}\right)} \frac{\Gamma(k+1)}{\Gamma(k-2 r+1)}= \\
& =\frac{1}{2^{2 r-1} \pi^{2}}(-1)^{r}\left[\Gamma\left(\frac{1}{2}-r\right)\right]^{4} \frac{\Gamma(k+1)}{\Gamma(k-2 r+1)} H(m, k, l) .
\end{aligned}
$$


Therefore

$$
\begin{aligned}
& R(m, n \mid l)= \\
& =\frac{2^{-2 r} \cos r \pi\left[\Gamma\left(\frac{1}{2}-r\right)\right]^{2}\left(l-r+\frac{1}{2}\right)}{\left[\Gamma\left(\frac{1}{2}\right)\right]^{2}} \frac{\Gamma(l+1)}{\Gamma(l-2 r+1)} \sum_{k=2 r}^{l+m} \frac{\Gamma(n-2 r+1) \Gamma(k+1)}{\Gamma(n+1) \Gamma(k-2 r+1)} a_{r}(k, n) H(m, k, l)= \\
& =2^{2 r-1} \pi^{2}(-1)^{r} \frac{1}{\left[\Gamma\left(\frac{1}{2}-r\right)\right]^{4}} \frac{2^{-2 r} \cos r \pi\left[\Gamma\left(\frac{1}{2}-r\right)\right]^{2}\left(l-r+\frac{1}{2}\right)}{\left[\Gamma\left(\frac{1}{2}\right)\right]^{2}} \frac{\Gamma(l+1)}{\Gamma(l-2 r+1)} \frac{\Gamma(n-2 r+1)}{\Gamma(n+1)} s(n)= \\
& =2^{-1} \pi \frac{\left(l-r+\frac{1}{2}\right)}{\left[\Gamma\left(\frac{1}{2}-r\right)\right]^{2}} \frac{\Gamma(l+1)}{\Gamma(l-2 r+1)} \frac{\Gamma(n-2 r+1)}{\Gamma(n+1)} s(n)= \\
& =\frac{1}{2} \frac{\pi \alpha_{\lambda}\left(\frac{l+m-n}{2}\right) \alpha_{\lambda}\left(\frac{l-m+n}{2}\right) \alpha_{\lambda}\left(\frac{l+m+n}{2}+\lambda\right) \alpha_{\lambda}\left(\frac{l-m-n}{2}-\lambda\right)}{\alpha_{\lambda}(l) \alpha_{\lambda}(l+\lambda)} .
\end{aligned}
$$

This expression is symmetric in $m$ and $n$, so that $c_{\frac{1}{2}-r}(m, n \mid l)=2 R(m, n \mid l)$. Thus, for $m, n, \lambda=\frac{1}{2}-r$ all satisfying the conditions stated above, and $l \stackrel{2}{=} m+n+2 \lambda+2 k, k$ a non-negative integer, the statements of Theorem 4.1 hold, and therefore this theorem holds generally by algebraic continuation as announced in Sect. 4 . 


\section{Expansion theorems}

Theorem 4.1 has been proved in Sect. 6, and it has been shown there that the conjectured identity (3.4) holds for the values of the parameters used in that section.

Returning to the conjectured identity (3.4), we consider the case when $m$ and $n$ are non-negative integers and $2 \lambda>0$ is an integer. The function $f(z)=\left(z^{2}-1\right)^{\lambda-\frac{1}{2}} D_{m}^{\lambda}(z) D_{n}^{\lambda}(z)$ satisfies the hypotheses of Theorem A.2 : note that $\left(z^{2}-1\right)^{\lambda-\frac{1}{2}}=z^{2 \lambda-1}\left(1-z^{-2}\right)^{\lambda-\frac{1}{2}}$ and that, at infinity, $f(z) \sim$ const. $z^{-(m+n+2 \lambda+1)}$. Therefore (3.4) holds and is uniformly convergent in any compact subset of $\mathbf{C} \backslash[-1,1]$. This uniform convergence allows the identification of the Laurent expansions of both sides of (3.4). Therefore $c_{\lambda}(m, n \mid l)$ can again be identified with the solution of the algebraic problem, and is given by Theorem 4.1. These conclusions can be assembled in the following theorem.

Theorem 7.1 Let $m$ and $n$ be non-negative integers, and suppose that one of the two following conditions is satisfied:

(i) $\lambda=\frac{1}{2}-r, r \geq 1$ is an integer such that $m \geq 2 r$ and $n \geq 2 r$;

(ii) $2 \lambda$ is a strictly positive integer;

Then

$$
D_{m}^{\lambda}(z) D_{n}^{\lambda}(z)=\sum_{\substack{l=m+n+2 \lambda+2 k \\ k \in \mathbf{Z}, \quad 0 \leq k}} c_{\lambda}(m, n \mid l) D_{l}^{\lambda}(z)
$$

holds with uniform convergence if $z$ remains in any compact subset of $\mathbf{C} \backslash[-1,1]$, with

$$
\begin{aligned}
c_{\lambda}(m, n \mid l) & =\frac{2^{2 \lambda-1}(l+\lambda) \Gamma(l+1) \Gamma(\lambda)^{2}}{i \pi^{2} \Gamma(l+2 \lambda)} \int_{\mathcal{C}}\left(t^{2}-1\right)^{\lambda-\frac{1}{2}} D_{m}^{\lambda}(t) D_{n}^{\lambda}(t) C_{l}^{\lambda}(t) d t \\
& =\frac{\pi \alpha_{\lambda}\left(\frac{l+m-n}{2}\right) \alpha_{\lambda}\left(\frac{l-m+n}{2}\right) \alpha_{\lambda}\left(\frac{l+m+n}{2}+\lambda\right) \alpha_{\lambda}\left(\frac{l-m-n}{2}-\lambda\right)}{\alpha_{\lambda}(l) \alpha_{\lambda}(l+\lambda)} .
\end{aligned}
$$

Here $\mathcal{C}$ may be taken as the circle $\{z \in \mathbf{C}:|z|=R\}, R>1$, traversed in the positive direction.

This theorem requires $m$ and $n$ to be integers. On the other hand Eqs (4.2) and (4.8) hold as identities between formal power series in $u$ and $v$, respectively (with $f_{k}(x, y, \eta)$ given by (4.11)) without such restrictions. Considering again the formal identity (4.8)

$$
\begin{aligned}
& F(x, 1-\eta ; x+\eta ; v) F(y, 1-\eta ; y+\eta ; v)= \\
& =\sum_{k=0}^{\infty} f_{k}(x, y, \eta) v^{k} F(x+y+2 k, 1-\eta ; x+y+\eta+2 k ; v)
\end{aligned}
$$

(with $f_{k}(x, y, \eta)$ given by (4.11)), we note that the common formal expansion in powers of $v$ on the lhs and the rhs is in fact convergent for $|v|<1$, since the lhs is holomorphic there. This does not imply that the series on the rhs converges. However if $a \geq 0, b \geq 0$ and $c>0$, all the coefficients of $F(a, b ; c ; v)$ as a power series in $v$ are positive, so that for $|v|<1,|F(a, b ; c ; v)| \leq F(a, b ; c ;|v|)$ and, for $0 \leq v<1, F(a, b ; c ; v)$ is the least upper bound of its partial sums. Let $x, y$ and $\eta$ be chosen such that

$$
x>0, \quad y>0, \quad x+y+2 \eta-1 \geq 0, \quad 1-\eta \geq 0, \quad x+\eta>0, \quad y+\eta>0, \quad x+y+\eta>0 .
$$

Then all the coefficients of the hypergeometric functions appearing in (7.4) as well as $f_{k}(x, y, \eta)$ are positive. We temporarily denote $G(v)$ the lhs of (7.4) and, for any integers $p \geq 0$ and $q \geq 0, S_{p}(v)$ the partial sum of the series on the rhs obtained by stopping at $k=p, G_{q}(v)$ the partial expansion of $G(v)$ in powers of $v$ up to the power $q, S_{p, q}(v)$ the expansion of $S_{p}(v)$ in powers of $v$ up to the power $q$. For $0 \leq v<1, G(v)=\sup _{q} G_{q}(v), S_{p}(v)=\sup _{q} S_{p, q}(v) \leq \sup _{q} G_{q}(v)=G(v)$. Thus $S_{p}(v)$ is bounded so that the series on the rhs converges. For $q \leq p, S_{p, q}(v)$ is equal to $G_{q}(v)$, so that 
$G_{q}(v) \leq S_{p}(v) \leq G(v)$, hence the sum of the series on the rhs is equal to $G(v)$. For $|v|<1$ and integer $p<p^{\prime},\left|S_{p^{\prime}}(v)-S_{p}(v)\right| \leq S_{p^{\prime}}(|v|)-S_{p}(|v|)$, hence the sequence $S_{p}(v)$ converges to a limit which is holomorphic in the unit disk and coincides with $G$ if $v=|v|$, hence is equal to $G$. Note also that $\left|S_{p}(v)\right| \leq G(|v|)$ for all $v$ in the unit disk. The map $z \mapsto v$ given by (3.8) maps $E_{+}(L) \cup\{\infty\}(L \geq 1$, see (2.16) $)$, onto the disk $\left\{v:|v|<L^{-2}\right\}$. In terms of the variables $m, n$ and $\lambda$, the conditions (7.5) follow from

$$
\lambda>0, \quad m+2 \lambda>0, \quad n+2 \lambda>0, \quad m+\lambda+1>0, \quad n+\lambda+1>0, \quad m+n+2 \lambda+1 \geq 0 .
$$

We thus obtain the following theorem:

Theorem 7.2 Under the conditions (7.6),

$$
D_{m}^{\lambda}(z) D_{n}^{\lambda}(z)=\sum_{\substack{l=m+n+2 \lambda+2 k \\ k \in \mathbf{Z}, \quad 0 \leq k}} c_{\lambda}(m, n \mid l) D_{l}^{\lambda}(z)
$$

holds as a convergent series for $z \in \mathbf{C} \backslash(-\infty, 1]$, with $c_{\lambda}(m, n \mid l)$ given by 7.3).

We emphasize that none of the parameters $m, n$ and $2 \lambda$ has to be an integer in this theorem, but the conditions (7.6) must be satisfied. The proof of this theorem can be slightly expanded to show that the convergence of (7.7) actually holds in the sense of functions with tempered behavior in $\mathbf{C} \backslash(-\infty, 1]$, so that the conclusion also holds for the boundary values of both sides in (7.7).

\subsection{Källén-Lehmann weights}

Returning to the $d$-dimensional Anti-de-Sitter space-time $X_{d}$ (or its covering $\widetilde{X}_{d}$ ), with $d \geq 2$, setting $\lambda=(d-1) / 2$, and taking into account the formulae [2.8, 2.9] and 2.12), we obtain the following results:

Theorem 7.3 Let $m$ and $n$ be integers satisfying the conditions 7.6 .

$$
W_{m+\frac{d-1}{2}}\left(z_{1}, z_{2}\right) W_{n+\frac{d-1}{2}}\left(z_{1}, z_{2}\right)=\sum_{\substack{l=m+n+d-1+2 k \\ k \in \mathbf{Z}, \quad 0 \leq k}} \rho(l ; m, n) W_{l+\frac{d-1}{2}}\left(z_{1}, z_{2}\right),
$$

with

$$
\begin{aligned}
\rho(l ; m, n) & =\frac{\Gamma(\lambda)}{2 \pi^{\lambda}} \frac{\alpha_{\lambda}\left(\frac{l+m-n}{2}\right) \alpha_{\lambda}\left(\frac{l-m+n}{2}\right) \alpha_{\lambda}\left(\frac{l+m+n}{2}+\lambda\right) \alpha_{\lambda}\left(\frac{l-m-n}{2}-\lambda\right)}{\alpha_{\lambda}(l) \alpha_{\lambda}(l+\lambda)}, \\
\lambda & =\frac{d-1}{2} .
\end{aligned}
$$

Here $z_{1} \in \mathcal{T}_{1-}, z_{1} \in \mathcal{T}_{1+}$, and the convergence holds in the sense of holomorphic functions with tempered behavior in $\mathcal{T}_{1-} \times \mathcal{T}_{1+}$, so that the above equation extends to the boundary values $\mathcal{W}$ of the functions $W$. The same equation holds in the case of $\widetilde{X}_{d}$, with $z_{1} \in \widetilde{\mathcal{T}}_{1-}$ and $z_{2} \in \widetilde{\mathcal{T}}_{1+}$, and with $m$ and $n$ not necessarily integers, but satisfying the conditions (7.6).

While $(l, m, n) \mapsto \rho(l ; m, n)$ will always denote the meromorphic function defined by (7.9), the sum in (7.8) begins at $l=m+n+d-1$. This spectral property is in sharp contrast to the situation in the de Sitter case. It reflects the fact that a genuine positive-energy condition has been imposed in the AdS case. 


\section{Some applications}

In this section the radius $R$ of $X_{d}$ will no longer be fixed as 1, and the AdS quadric with radius $R$ given by (2.1) will be denoted $X_{d}(R)$. In this case, for the free Klein-Gordon field $\phi$ labelled by $n+(d-1) / 2$,

$$
\begin{aligned}
& \left(\Omega, \phi\left(x_{1}\right) \phi\left(x_{2}\right) \Omega\right)=\mathcal{W}_{n+\frac{d-1}{2}}\left(x_{1}, x_{2}\right)=\lim _{\substack{z_{1} \in \mathcal{T}_{1-}, z_{1} \rightarrow x_{1}, \quad z_{2} \in \mathcal{T}_{1+}}} W_{n+\frac{d-1}{2}}\left(z_{1}, z_{2}\right), \\
& W_{n+\frac{d-1}{2}}\left(z_{1}, z_{2}\right)=R^{2-d} w_{n+\frac{d-1}{2}}\left(\frac{z_{1} \cdot z_{2}}{R^{2}}\right),
\end{aligned}
$$

where $w_{n+(d-1) / 2}$ is given by (2.12). We keep the formula (7.9) so that the rsh of (7.8) now acquires a factor $R^{2-d}$.

In a Minkowski, de Sitter or Anti-de-Sitter space, we consider three commuting Klein-Gordon fields $\phi_{0}, \phi_{1}$ and $\phi_{2}$ operating in the same Fock space $\mathcal{F}$ (with vacuum $\Omega$ ), and denote $\mathcal{L}(x)=\phi_{0}(x) \phi_{1}(x) \phi_{2}(x)$. The fields have masses $m_{j}$ or, in the AdS case, parameters $n_{j}+(d-1) / 2, j=0,1,2$. Let $f_{0}$ be a test-function and $\psi_{0}=\int f_{0}(x) \phi_{0}(x) \Omega d x$. Let $E_{1,2}$ be the projector on the subspace spanned by the states of the form $\int \varphi\left(x_{1}, x_{2}\right) \phi_{1}\left(x_{1}\right) \phi_{2}\left(x_{2}\right) \Omega d x_{1} d x_{2}$. If an interaction of the form $I_{g}=\int \gamma g(x) \mathcal{L}(x) d x$ is introduced, with a coupling constant $\gamma$, and with $g$ a real, rapidly decreasing, smooth switching-off factor, the lowest order transition probability from $\psi_{0}$ to any state in $E_{1,2} \mathcal{F}$ is given by

$$
\begin{aligned}
& \frac{\left(\psi_{0}, I_{g} E_{1,2} I_{g} \psi_{0}\right)}{\left(\psi_{0}, \psi_{0}\right)}= \\
& =\frac{\gamma^{2} \int \overline{f_{0}(x)} g(u) g(v) f_{0}(y) \mathcal{W}_{m_{0}}(x, u) \mathcal{W}_{m_{1}}(u, v) \mathcal{W}_{m_{2}}(u, v) \mathcal{W}_{m_{0}}(v, y) d x d u d v d y}{\int \overline{f_{0}(x)} \mathcal{W}_{m_{0}}(x, y) f_{0}(y) d x d y} .
\end{aligned}
$$

Attempting to take the "adiabatic limit" of this expression, i.e. its limit as $g$ tends to 1 , leads, in Minkowski or de Sitter space-time, to a divergence for which the traditional remedy is the Fermi golden rule. This requires involved computations in the de Sitter case [4, 3]. It will be seen below that the corresponding calculation is considerably easier in the case of the AdS space-time. The question of its physical interpretation is, however, considerably more difficult. It seems nevertheless worth giving it here as a simple application of Theorem 7.3 Another ingredient is the "projector identity" for $X_{d}(R)$ (analogous to a similar property in the Minkowskian and de Sitter case [4]), given by the following theorem.

Theorem 8.1 (Projector identity) Let $n_{1}, n_{2}$ and $d$ be integers satisfying $d \geq 2, n_{1}+d-1>0$, $n_{2}+d-1>0$ and $n_{1}+n_{2}+d-1>0$. Then

$$
\int_{X_{d}} W_{n_{1}+\frac{d-1}{2}}\left(z_{1}, u\right) W_{n_{2}+\frac{d-1}{2}}\left(u, z_{2}\right) d u=\frac{2 \pi R^{2}}{\left(2 n_{1}+d-1\right)} \delta_{n_{1} n_{2}} W_{n_{1}+\frac{d-1}{2}}\left(z_{1}, z_{2}\right),
$$

Here $z_{1} \in \mathcal{T}_{-}$and $z_{2} \in \mathcal{T}_{+}$, and du denotes the standard invariant measure on $X_{d}$, $d u=2 R \delta(u \cdot u-$ $\left.R^{2}\right) d u^{0} \ldots d u^{d}$. The convergence is absolute and uniform when $\left(z_{1}, z_{2}\right)$ remains in a compact subset of $\mathcal{T}_{-} \times \mathcal{T}_{+}$, and, in fact the convergence takes place in the space of functions holomorphic with tempered behavior in $\mathcal{T}_{-} \times \mathcal{T}_{+}$, and the equation continues to hold for the boundary values $\mathcal{W}_{n_{j}+\frac{d-1}{2}}$ of the functions $W_{n_{j}+\frac{d-1}{2}}$.

This theorem is proved in Appendix D Note that it gives another proof of the positive-definiteness of $W_{n+\frac{d-1}{2}}\left(z_{1}, z_{2}\right)$ for integer $n$ satisfying $2 n+d-1>0$.

Let $n_{0}, n_{1}$, and $n_{2}$ be integers such that

$$
\begin{array}{ll}
n_{1}+d-1>0, & n_{2}+d-1>0, \quad n_{1}+\frac{d-1}{2}+1>0, \quad n_{2}+\frac{d-1}{2}+1>0, \\
n_{0}+d-1>0, & n_{0}+n_{1}+n_{2}+2(d-1)>0 .
\end{array}
$$


(8.5) implies $n_{1}+n_{2}+d+1>0$ hence $n_{1}+n_{2}+d \geq 0$.) Let $z_{1} \in \mathcal{T}_{1-}, z_{2} \in \mathcal{T}_{1+}, g_{1}$ and $g_{2}$ be two smooth functions with rapid decrease on $X_{d}$. By Theorem 7.3

$$
\begin{aligned}
& \int_{X_{d} \times X_{d}} W_{n_{0}+\frac{d-1}{2}}\left(z_{1}, u\right) W_{n_{1}+\frac{d-1}{2}}(u, v) W_{n_{2}+\frac{d-1}{2}}(u, v) W_{n_{0}+\frac{d-1}{2}}\left(v, z_{2}\right) g_{1}(u) d u g_{2}(v) d v= \\
& =\sum_{\substack{l=n_{1}+n_{2}+d-1+2 k \\
k \in \mathbf{Z}, \quad 0 \leq k}} R^{2-d} \rho\left(l ; n_{1}, n_{2}\right) \times \\
& \times \int_{X_{d} \times X_{d}} W_{n_{0}+\frac{d-1}{2}}\left(z_{1}, u\right) \mathcal{W}_{l+\frac{d-1}{2}}(u, v) W_{n_{0}+\frac{d-1}{2}}\left(v, z_{2}\right) g_{1}(u) d u g_{2}(v) d v .
\end{aligned}
$$

Note that by arguments similar to the proof of Theorem 8.1 the integral in the lhs of (8.7) is absolutely convergent even if $g_{1}$ and $g_{2}$ are set equal to 1 . In the last integral, we can let $g_{1}$ tend to 1 and execute the integration over $u$ by applying the projector identity, then let $g_{2}$ tend to 1 and similarly execute the integration over $v$. Thus

$$
\begin{array}{r}
\int_{X_{d} \times X_{d}} W_{n_{0}+\frac{d-1}{2}}\left(z_{1}, u\right) W_{n_{1}+\frac{d-1}{2}}(u, v) W_{n_{2}+\frac{d-1}{2}}(u, v) W_{n_{0}+\frac{d-1}{2}}\left(v, z_{2}\right) d u d v= \\
=R^{6-d}\left(\frac{2 \pi}{2 n_{0}+d-1}\right)^{2} \rho\left(n_{0} ; n_{1}, n_{2}\right) W_{n_{0}+\frac{d-1}{2}}\left(z_{1}, z_{2}\right)
\end{array}
$$

provided $n_{0}-n_{1}-n_{2}-d+1$ is an even non-negative integer, the lhs being otherwise equal to zero. Under the same condition the limit as $g$ tends to 1 of (8.3) is given by

$$
\text { Prob. }\left(\psi_{0} \rightarrow n_{1}, n_{2}\right) \stackrel{\text { def }}{=} \lim _{g \rightarrow 1} \frac{\left(\psi_{0}, I_{g} E_{1,2} I_{g} \psi_{0}\right)}{\left(\psi_{0}, \psi_{0}\right)}=R^{6-d}\left(\frac{2 \pi \gamma}{2 n_{0}+d-1}\right)^{2} \rho\left(n_{0} ; n_{1}, n_{2}\right) \text {. }
$$

As in the de Sitter case, this expression is independent of the initial wave-function $f_{0}$. There has been no necessity for using the Fermi golden rule. In order do so nevertheless, i.e. take the "time-average" of this "transition probability", we need to divide the expression in (8.9) by some plausible "total time" of the form $K_{0} R$. The result is

$$
\text { "Time-average" (Prob. } \left.\left(\psi_{0} \rightarrow n_{1}, n_{2}\right)\right)=\frac{4 \pi^{2} R^{5-d} \gamma^{2}}{K_{0}\left(2 n_{0}+d-1\right)^{2}} \rho\left(n_{0} ; n_{1}, n_{2}\right) .
$$

\subsection{Minkowskian limits}

In this subsection, $n$ will not necessarily be an integer and $w_{n+(d-1) / 2}$ is regarded as holomorphic in $\mathbf{C} \backslash(-\infty, 1]$.

If the origin of coordinates in $\mathbf{R}^{d+1}$ is transported to the point $R e_{d}=(0, \ldots, R) \in X_{d}(R)$, and the radius $R$ is allowed to tend to $+\infty$, the translated quadric $X_{d}(R)-R e_{d}$ tends to the Minkowski subspace $M_{d}=\left\{x: x^{d}=0\right\}$. The Klein-Gordon field on $X_{d}(R)-R e_{d}$ with parameter $n=m R>0$ can be considered to tend to the Klein-Gordon field on $M_{d}$. Let indeed

$$
z_{1}=R e_{d}, \quad z_{2}=R \sin (t / R) e_{0}+R \cos (t / R) e_{d}, \quad \operatorname{Im} t>0
$$

It can be shown that

$$
\begin{aligned}
\lim _{R \rightarrow+\infty} R^{2-d} w_{m R+\frac{d-1}{2}}\left(\frac{z_{1} \cdot z_{2}}{R^{2}}\right) & =\frac{i^{d-1}}{4(2 \pi)^{\frac{d}{2}-1}} m^{d-2}(m t)^{1-\frac{d}{2}} H_{\frac{d}{2}-1}^{(1)}(m t) \\
& =\widehat{w} \text { Minkowski, } m\left(t^{2}\right) .
\end{aligned}
$$


Here $\widehat{w}$ Minkowski, $m$ is holomorphic in $\mathbf{C} \backslash \mathbf{R}_{+}$and the free Klein-Gordon field $\phi_{\text {Minkowski, } m}$ of mass $m$ on $M_{d}$ satisfies

$$
\left(\Omega, \phi \text { Minkowski, } m\left(r_{1}\right) \phi \text { Minkowski, } m\left(r_{2}\right) \Omega\right)=\underset{\substack{\operatorname{Im} u_{1} \in V_{-}, u_{1} \rightarrow r_{1},}}{\lim u_{2} \rightarrow r_{2}} \underset{u_{+}}{ } \widehat{w} \text { Minkowski, } m\left(\left(u_{1}-u_{2}\right)^{2}\right) .
$$

It is therefore interesting to consider the behavior of the Källén-Lehmann weight (7.9) and the expression (8.9) in the same limit, as it was done in [4, 3] in the de Sitter case. By Stirling's formula, as Re $t \rightarrow+\infty$ at fixed $x, y$, and $\lambda$,

$$
\frac{\Gamma(t+x)}{\Gamma(t+y)} \sim t^{x-y}, \quad \alpha_{\lambda}(t) \sim \frac{t^{\lambda-1}}{\Gamma(\lambda)} .
$$

For fixed $m_{0}>0, m_{1}>0$, and $m_{2}>0, \lambda=(d-1) / 2$, we have therefore

$$
\begin{aligned}
\lim _{R \rightarrow+\infty} R^{3-d} & \rho\left(R m_{0} ; R m_{1}, R m_{2}\right)=\theta\left(m_{0}-m_{1}-m_{2}\right) \frac{1}{2^{2 d-5} \pi^{\frac{d-1}{2}} m_{0}^{d-3} \Gamma\left(\frac{d-1}{2}\right)} \times \\
\times & {\left[\left(m_{0}-m_{1}+m_{2}\right)\left(m_{0}+m_{1}-m_{2}\right)\left(m_{0}+m_{1}+m_{2}\right)\left(m_{0}-m_{1}-m_{2}\right)\right]^{\frac{d-3}{2}} } \\
& =4 m_{0} \rho_{\operatorname{Min}}\left(m_{0}^{2} ; m_{1}, m_{2}\right) .
\end{aligned}
$$

If we set similarly $n_{j}=R m_{j}$ in (8.10), this expression tends, as $R \rightarrow+\infty$, to

$$
\frac{4 \pi^{2} \gamma^{2}}{K_{0} m_{0}} \rho_{\text {Min }}\left(m_{0}^{2} ; m_{1}, m_{2}\right) .
$$

With the choice $K_{0}=4 \pi,(8.16)$ becomes equal to an analogous quantity in Minkowski QFT, i.e. the inverse lifetime of a particle of mass $m_{0}$ decaying into two particles of masses $m_{1}$ and $m_{2}$ in its rest-frame (see [4, 3]). 


\section{A Appendix. Jacobi and ultraspherical functions}

\section{A.1 Jacobi polynomials and functions of the second kind}

The major part of this and the next subsections is taken from [17. In both subsections, $n \geq 0$ is an integer. For arbitrary complex $\alpha$ and $\beta$, the Jacobi polynomial $P_{n}^{(\alpha, \beta)}$ is given by

$$
\begin{aligned}
P_{n}^{(\alpha, \beta)}(x) & =\left(\begin{array}{c}
n+\alpha \\
n
\end{array}\right) F\left(-n, n+\alpha+\beta+1 ; \alpha+1 ; \frac{1-x}{2}\right)= \\
& =\frac{1}{n !} \sum_{p=0}^{n}\left(\begin{array}{c}
n \\
p
\end{array}\right)(n+\alpha+\beta+1) \ldots(n+\alpha+\beta+p) \times \\
& \times(\alpha+p+1) \ldots(\alpha+n)\left(\frac{x-1}{2}\right)^{p} .
\end{aligned}
$$

([17, (4.21.2), p. 62]). By definition,

$$
\left(\begin{array}{l}
a \\
b
\end{array}\right)=\frac{\Gamma(a+1)}{\Gamma(b+1) \Gamma(a-b+1)}
$$

Thus $P_{n}^{(\alpha, \beta)}(x)$ is a polynomial in $x, \alpha$ and $\beta$. Rodrigues' formula ([17, (4.3.1), p. 67]),

$$
(1-x)^{\alpha}(1+x)^{\beta} P_{n}^{(\alpha, \beta)}(x)=\frac{(-1)^{n}}{2^{n} n !}\left(\frac{d}{d x}\right)^{n}(1-x)^{n+\alpha}(1+x)^{n+\beta},
$$

may be taken as another definition. For $\operatorname{Re}(\alpha+n)>-1$ and $\operatorname{Re}(\beta+n)>-1$, and excluding the case $n=0$ and $\alpha+\beta+1=0$, the Jacobi function of the second kind $Q_{n}^{(\alpha, \beta)}(z)$ is given by

$$
\begin{aligned}
(z-1)^{\alpha}(z+1)^{\beta} Q_{n}^{(\alpha, \beta)}(z) & = \\
& 2^{-n-1} \int_{-1}^{1}(1-t)^{n+\alpha}(1+t)^{n+\beta}(z-t)^{-n-1} d t
\end{aligned}
$$

$Q_{n}^{(\alpha, \beta)}$ extends as a function holomorphic in $\mathbf{C} \backslash(-\infty, 1]$, and $Q_{n}^{(\alpha, \beta)}(z) \sim z^{-n-\alpha-\beta-1}$ as $z \rightarrow \infty$. ([17, (4.61.1), p. 73]). Another representation is ([17, p. 74]) :

$$
\begin{aligned}
Q_{n}^{(\alpha, \beta)}(z) & =2^{n+\alpha+\beta} \frac{\Gamma(n+\alpha+1) \Gamma(n+\beta+1)}{\Gamma(2 n+\alpha+\beta+2)}(z-1)^{-n-\alpha-1}(z+1)^{-\beta} \times \\
& \times F\left(n+\alpha+1, n+1 ; 2 n+\alpha+\beta+2 ; \frac{2}{1-z}\right)
\end{aligned}
$$

Eq. A.5 is derived from Eq (A.4) and they both provide the same analytic extension to complex values of $n, \alpha$ and $\beta$.

\section{A.2 Ultraspherical functions}

For a positive integer $n$ and a complex $\lambda$, the Gegenbauer polynomial $C_{n}^{\lambda} \equiv P_{n}^{(\lambda)}$ is defined as

$$
C_{n}^{\lambda}(x)=U(n, \lambda) P_{n}^{\left(\lambda-\frac{1}{2}, \lambda-\frac{1}{2}\right)}(x)
$$

with

$$
U(n, \lambda)=\frac{\Gamma\left(\lambda+\frac{1}{2}\right) \Gamma(n+2 \lambda)}{\Gamma(2 \lambda) \Gamma\left(n+\lambda+\frac{1}{2}\right)}=\frac{2^{1-2 \lambda} \pi^{1 / 2} \Gamma(n+2 \lambda)}{\Gamma(\lambda) \Gamma\left(n+\lambda+\frac{1}{2}\right)} .
$$


Hence

$$
\begin{aligned}
C_{n}^{\lambda}(x) & =\frac{\Gamma(n+2 \lambda)}{\Gamma(n+1) \Gamma(2 \lambda)} F\left(-n, n+2 \lambda ; \lambda+\frac{1}{2} ; \frac{1-x}{2}\right) \\
& =\frac{2^{\frac{1}{2}-\lambda} \Gamma\left(\frac{1}{2}\right) \Gamma(n+2 \lambda)\left(x^{2}-1\right)^{\frac{1}{4}-\frac{\lambda}{2}}}{\Gamma(n+1) \Gamma(\lambda)} P_{n-\frac{1}{2}+\lambda}^{\frac{1}{2}-\lambda}(x) .
\end{aligned}
$$

Here $P_{\nu}^{\mu}$ is the Legendre function ([15, $3.2(3)$ p. 122]). Rodrigues's formula gives

$$
C_{n}^{\lambda}(x)=\frac{2^{1-2 \lambda} \pi^{1 / 2} \Gamma(n+2 \lambda)(-1)^{n}}{\Gamma(\lambda) \Gamma\left(n+\frac{1}{2}+\lambda\right) 2^{n} n !}\left(1-x^{2}\right)^{\frac{1}{2}-\lambda}\left(\frac{d}{d x}\right)^{n}\left(1-x^{2}\right)^{n+\lambda-\frac{1}{2}} .
$$

The Gegenbauer polynomials have a generating function ([15, 3.15.1(1), p. 175], [17, p. 83]) :

$$
\sum_{n=0}^{\infty} C_{n}^{\lambda}(x) h^{n}=\left(1-2 h x+h^{2}\right)^{-\lambda}
$$

This shows that $\lambda \mapsto C_{n}^{\lambda}(x)$ is entire.

Define

$$
D_{n}^{\lambda}(z)=U(n, \lambda) Q_{n}^{\left(\lambda-\frac{1}{2}, \lambda-\frac{1}{2}\right)}(z) .
$$

$\left(z^{2}-1\right)^{\lambda-\frac{1}{2}} D_{n}^{\lambda}(z)$ extends to a function holomorphic in $\mathbf{C} \backslash[-1,1]$. As a special case of (A.4), for $z$ in this cut-plane, and supposing $\operatorname{Re}(n+2 \lambda)>0$ (which implies $\operatorname{Re}\left(n+\lambda-\frac{1}{2}\right)>-1$ for $n \geq 0$ ),

$$
\begin{aligned}
\left(z^{2}-1\right)^{\lambda-\frac{1}{2}} D_{n}^{\lambda}(z) & =\frac{(-2)^{-n-1} 2^{1-2 \lambda} \pi^{1 / 2} \Gamma(n+2 \lambda)}{\Gamma(\lambda) \Gamma\left(n+\lambda+\frac{1}{2}\right)} \int_{-1}^{1}\left(1-t^{2}\right)^{n+\lambda-\frac{1}{2}}(t-z)^{-n-1} d t \\
& =\frac{(-2)^{-n-1} 2^{1-2 \lambda} \pi^{1 / 2} \Gamma(n+2 \lambda)}{n ! \Gamma(\lambda) \Gamma\left(n+\lambda+\frac{1}{2}\right)}\left(\frac{d}{d z}\right)^{n} \int_{-1}^{1}\left(1-t^{2}\right)^{n+\lambda-\frac{1}{2}}(t-z)^{-1} d t .
\end{aligned}
$$

Therefore, in the sense of tempered distributions, on the real axis,

$$
\begin{aligned}
& \operatorname{disc}\left[\left(x^{2}-1\right)^{\lambda-\frac{1}{2}} D_{n}^{\lambda}(x)\right]=\frac{2 i \pi(-2)^{-n-1} 2^{1-2 \lambda} \pi^{1 / 2} \Gamma(n+2 \lambda)}{n ! \Gamma(\lambda) \Gamma\left(n+\lambda+\frac{1}{2}\right)} \times \\
& \times\left(\frac{d}{d x}\right)^{n}\left[\theta(x+1) \theta(1-x)\left(1-x^{2}\right)^{n+\lambda-\frac{1}{2}}\right] .
\end{aligned}
$$

(A.5) gives, together with [15, 3.2 (37) p. 132], and [15, 3.3.1 (2) p. 140],

$$
\begin{aligned}
D_{n}^{\lambda}(x) & =\frac{2^{\frac{1}{2}-\lambda} \pi^{1 / 2} \Gamma(n+2 \lambda)\left(x^{2}-1\right)^{\frac{1}{4}-\frac{\lambda}{2}}}{\Gamma(\lambda) \Gamma(n+1)} e^{i \pi\left(\lambda-\frac{1}{2}\right)} Q_{n+\lambda-\frac{1}{2}}^{\frac{1}{2}-\lambda}(x) \\
& =\frac{2^{\frac{1}{2}-\lambda} \pi^{1 / 2}\left(x^{2}-1\right)^{\frac{1}{4}-\frac{\lambda}{2}}}{\Gamma(\lambda)} e^{i \pi\left(\frac{1}{2}-\lambda\right)} Q_{n+\lambda-\frac{1}{2}}^{\lambda-\frac{1}{2}}(x) .
\end{aligned}
$$

Eq. A.16) and [15, 3.2 (8) p. 122], or Eq. (A.17) and [15, 3.2 (5) p. 122], give

$$
D_{n}^{\lambda}(x)=\frac{\pi \Gamma(n+2 \lambda)}{\Gamma(\lambda) \Gamma(n+\lambda+1)}(2 x)^{-n-2 \lambda} F\left(\frac{n+2 \lambda}{2}, \frac{n+2 \lambda+1}{2} ; n+\lambda+1 ; \frac{1}{x^{2}}\right) .
$$

This formula, (A.13), (A.16), (A.17), and (2.14) all provide the same analytic extension of $D_{n}^{\lambda}$ to complex values of $n$.

The Legendre function of the second kind "on the cut", i.e. on $(-1,1)$, are defined as follows:

$$
\mathbf{Q}_{\nu}^{\mu}(x)=\frac{1}{2} e^{-i \pi \mu}\left[e^{-\frac{i \pi \mu}{2}} Q_{\nu}^{\mu}(x+i 0)+e^{\frac{i \pi \mu}{2}} Q_{\nu}^{\mu}(x-i 0)\right]
$$


It follows that

$$
\begin{aligned}
\mathbf{D}_{n}^{\lambda}(x) & =\frac{1}{2}\left[D_{n}^{\lambda}(x+i 0)+D_{n}^{\lambda}(x-i 0)\right]= \\
& =\frac{\pi^{1 / 2}}{2^{\lambda+\frac{1}{2}} \Gamma(\lambda)}\left(1-x^{2}\right)^{\frac{1}{4}-\frac{\lambda}{2}} e^{-i \pi\left(\lambda-\frac{1}{2}\right)}\left[e^{-i \pi\left(\frac{\lambda}{2}-\frac{1}{4}\right)} Q_{n+\lambda-\frac{1}{2}}^{\lambda-\frac{1}{2}}(x+i 0)+e^{i \pi\left(\frac{\lambda}{2}-\frac{1}{4}\right)} Q_{n+\lambda-\frac{1}{2}}^{\lambda-\frac{1}{2}}(x-i 0)\right] \\
& =\frac{\pi^{1 / 2}}{2^{\lambda-\frac{1}{2}} \Gamma(\lambda)}\left(1-x^{2}\right)^{\frac{1}{4}-\frac{\lambda}{2}} \mathbf{Q}_{n+\lambda-\frac{1}{2}}^{\lambda-\frac{1}{2}}(x)
\end{aligned}
$$

Also note the following formulae from [15, p. 143], valid for $-1<x<1$ :

$$
\begin{gathered}
\mathbf{P}_{\nu}^{\mu}(x)=e^{\frac{i \pi \mu}{2}} P_{\nu}^{\mu}(x+i 0)=e^{\frac{-i \pi \mu}{2}} P_{\nu}^{\mu}(x-i 0)= \\
=\frac{1}{\Gamma(1-\mu)}\left(\frac{1+x}{1-x}\right)^{\frac{\mu}{2}} F\left(-\nu, \nu+1 ; 1-\mu ; \frac{1-x}{2}\right) . \\
\Gamma(\nu+\mu+1) \mathbf{Q}_{\nu}^{-\mu}(x)=\Gamma(\nu-\mu+1)\left[\mathbf{Q}_{\nu}^{\mu}(x) \cos (\mu \pi)+\frac{\pi}{2} \mathbf{P}_{\nu}^{\mu}(x) \sin (\mu \pi)\right] .
\end{gathered}
$$

Thus it follows from (A.20) and (A.22) that

$$
\mathbf{D}_{n}^{\lambda}(x)=\frac{\pi^{1 / 2} \Gamma(n+2 \lambda)\left(1-x^{2}\right)^{\frac{1}{4}-\frac{\lambda}{2}}}{2^{\lambda-\frac{1}{2}} \Gamma(n+1) \Gamma(\lambda)}\left[\mathbf{Q}_{n+\lambda-\frac{1}{2}}^{\frac{1}{2}-\lambda}(x) \cos \left(\pi\left(\frac{1}{2}-\lambda\right)\right)+\frac{\pi}{2} \mathbf{P}_{n+\lambda-\frac{1}{2}}^{\frac{1}{2}-\lambda}(x) \sin \left(\pi\left(\frac{1}{2}-\lambda\right)\right)\right]
$$

\section{A.3 The special case of $\lambda=\frac{1}{2}-r$ with integer $r \geq 0$}

In this subsection, $r$ and $n$ are integers such that $0 \leq 2 r \leq n$. Eq. (A.10) takes the form

$$
C_{n}^{\frac{1}{2}-r}(x)=\frac{(-2)^{-n}}{n !} U\left(n, \frac{1}{2}-r\right)\left(1-x^{2}\right)^{r}\left(\frac{d}{d x}\right)^{n}\left(1-x^{2}\right)^{n-r} .
$$

$U(n, \lambda)$ has been defined in (A.7). Since $n-r \geq 0$, this displays the fact that the polynomial $C_{n}^{\frac{1}{2}-r}(x)$ is divisible by $\left(1-x^{2}\right)^{r}$.

If $F$ is a holomorphic function of tempered behavior in the complement of the real axis, and $\varphi$ a function holomorphic in a complex neighborhood of the real axis, then

$$
\varphi(x) \operatorname{disc} F(x)=\operatorname{disc}[\varphi(x) F(x)] .
$$

Applying this to $\varphi(x)=\left(x^{2}-1\right)^{r}$ and $F(x)=\left(x^{2}-1\right)^{-r} D_{n}^{\frac{1}{2}-r}(x)$, we obtain from (A.15)

$$
\operatorname{disc} D_{n}^{\frac{1}{2}-r}(x)=\frac{2 i \pi(-2)^{-n-1}}{n !} U\left(n, \frac{1}{2}-r\right)\left(x^{2}-1\right)^{r}\left(\frac{d}{d x}\right)^{n}\left[\theta(x+1) \theta(1-x)\left(1-x^{2}\right)^{n-r}\right] .
$$

This can be rewritten as

$$
\begin{aligned}
\operatorname{disc} D_{n}^{\frac{1}{2}-r}(x) & =\theta(x+1) \theta(1-x) \frac{(-i \pi)(-2)^{-n}}{n !} U\left(n, \frac{1}{2}-r\right)\left(x^{2}-1\right)^{r}\left(\frac{d}{d x}\right)^{n}\left(1-x^{2}\right)^{n-r}= \\
& =(-i \pi)(-1)^{r} \theta(x+1) \theta(1-x) C_{n}^{\frac{1}{2}-r}(x) .
\end{aligned}
$$

To see that (A.27) follows from (A.26), we first note that, by Leibniz's rule,

$$
\begin{aligned}
&\left(1-x^{2}\right)^{r}\left(\frac{d}{d x}\right)^{n}\left[\theta(x+1) \theta(1-x)\left(1-x^{2}\right)^{n-r}\right]= \\
&\left.u^{r} v^{r}\left(\frac{\partial}{\partial u}-\frac{\partial}{\partial v}\right)^{n}\left[\theta(u) u^{n-r} \theta(v) v^{n-r}\right]\right|_{u=x+1, v=1-x}
\end{aligned}
$$


It is then easy to check that if $s$ is an integer such that $0 \leq s \leq n$,

$$
u^{r}(d / d u)^{s} \theta(u) u^{n-r}=\theta(u) u^{r}(d / d u)^{s} u^{n-r} .
$$

As a consequence, if $z \in \mathbf{C} \backslash[-1,1]$,

$$
D_{n}^{\frac{1}{2}-r}(z)=\frac{(-1)^{r+1}}{2} \int_{-1}^{1} \frac{C_{n}^{\frac{1}{2}-r}(t)}{t-z} d t
$$

Indeed both sides have the same discontinuity and vanish at infinity. This can be rewritten (see [17, p. 77]) as

$$
D_{n}^{\frac{1}{2}-r}(z)=\frac{(-1)^{r+1}}{2} \int_{-1}^{1} \frac{C_{n}^{\frac{1}{2}-r}(t)-C_{n}^{\frac{1}{2}-r}(z)}{t-z} d t+\frac{(-1)^{r+1} C_{n}^{\frac{1}{2}-r}(z)}{2} \int_{-1}^{1} \frac{d t}{t-z} .
$$

The first term is a polynomial of degree $n-1$ in $z$. The second is equal to

$$
\frac{(-1)^{r+1} C_{n}^{\frac{1}{2}-r}(z)}{2} \log \left(\frac{z-1}{z+1}\right) \text {. }
$$

Since $C_{n}^{\frac{1}{2}-r}(z)$ is divisible by $\left(z^{2}-1\right)^{r}$ this shows that the boundary values of $D_{n}^{\frac{1}{2}-r}(z)$ on the real axis are continuous if $r \geq 1$, and in fact belong to $\mathcal{C}^{r-1}$.

Formulae (A.20) and (A.23) become:

$$
\begin{aligned}
\mathbf{D}_{n}^{\frac{1}{2}-r}(x) & =\frac{2^{r} \pi^{1 / 2}\left(1-x^{2}\right)^{r / 2}}{\Gamma\left(\frac{1}{2}-r\right)} \mathbf{Q}_{n-r}^{-r}(x) \\
& =\frac{2^{r} \pi^{1 / 2} \Gamma(n-2 r+1)\left(1-x^{2}\right)^{r / 2}}{\Gamma(n+1) \Gamma\left(\frac{1}{2}-r\right)} \mathbf{Q}_{n-r}^{r}(x) \cos (\pi r) .
\end{aligned}
$$

\section{A.4 Expansion of holomorphic functions in terms of ultraspherical functions}

Expansions of holomorphic functions in series of Legendre polynomials and functions of the second kind are classical. Recall that the Legendre polynomials $P_{n}=P_{n}^{(0,0)}$ and Legendre functions of the second kind $Q_{n}=Q_{n}^{(0,0)}$ are given by

$$
P_{n}(z)=\frac{1}{2^{n} n !}\left(\frac{d}{d z}\right)^{n}\left(z^{2}-1\right)^{n}, \quad Q_{n}(z)=-\frac{1}{2} \int_{-1}^{1} \frac{P_{n}(t)}{t-z} d t
$$

The following theorem is classical (the notations $E(L), E_{ \pm}(L)$ have been defined in Sect. 2).

Theorem A.1 Let $F$ be holomorphic at infinity, with $F(\infty)=0$. Then

$$
F(z)=\sum_{n=0}^{\infty} b_{n} Q_{n}(z)
$$

with

$$
b_{n}=\frac{2 n+1}{2 i \pi} \int_{\mathcal{C}} F(z) P_{n}(z) d z .
$$

The contour $\mathcal{C}$ may be taken to be any $E(L)$ such that $F$ is holomorphic in $E_{+}(L-\varepsilon), L>L-\varepsilon>1$. The expansion (A.37) converges uniformly on any compact subset of the exterior of the smallest ellipse $E\left(L_{0}\right)$ in the exterior of which $F$ is regular.

This theorem has a generalization to Jacobi polynomials and functions of the second kind: see Theorems 9.2.1 and 9.2.2 in [17, pp. 251-252]. Applying the second of these theorems to the special case of ultraspherical functions yields: 
Theorem A.2 Assume $\lambda>0$. Let $f$ be holomorphic in a neighborhood of $\infty$, with $f(\infty)=0$. Then

$$
f(y)=\left(y^{2}-1\right)^{\lambda-\frac{1}{2}} \sum_{n=0}^{\infty} b_{n} D_{n}^{\lambda}(y) .
$$

This expansion is convergent in the exterior of the smallest ellipse with foci \pm 1 in the exterior of which $f$ is holomorphic. The sum of the semi-axes of this ellipse is

$$
\limsup _{n \rightarrow \infty}\left|b_{n}\right|^{1 / n} .
$$

The coefficients $b_{n}$ are given by

$$
b_{n}=\frac{2^{2 \lambda-1}(n+\lambda) \Gamma(n+1) \Gamma(\lambda)^{2}}{i \pi^{2} \Gamma(n+2 \lambda)} \int f(x) C_{n}^{\lambda}(x) d x,
$$

where the integral is over any larger ellipse.

As stated here, this theorem does not apply to the case $\lambda=\frac{1}{2}-r, r \in \mathbf{N}$ which we will need to consider. Although it would be possible to extend the proof of Theorem A.2 at the cost of some effort, we will rely on an elementary application of Theorem A.1 which will suffice for our needs.

Let $F$ be a function holomorphic in $\mathbf{C} \backslash[-1,1]$ and at infinity, i.e. having a convergent Laurent expansion

$$
F(z)=\sum_{n=0}^{\infty} c_{n} z^{-n}, \quad c_{n}=\frac{1}{2 i \pi} \int_{\mathcal{C}} z^{n-1} F(z) d z .
$$

We suppose that for a certain integer $N>1, c_{n}$ vanishes for all $n<N$, i.e. the Laurent series starts at $n=N$. In eq. A.42 the contour $\mathcal{C}$ may be the circle $\{z:|z|=R\}$, with $R>1$, with the positive orientation, or any smooth closed contour homotopic to this circle in the cut-plane $\mathbf{C} \backslash[-1,1]$. We can define, for each integer $r$ with $0 \leq r<N$

$$
F^{(-r)}(z)=\sum_{n=N}^{\infty} \frac{(-1)^{r} c_{n}}{(n-1) \ldots(n-r) z^{n-r}} .
$$

Thus $F^{(0)}=F$ and, if $r>0$,

$$
F^{(-r)}(z)=\int_{\infty}^{z} F^{(-r+1)}(t) d t
$$

the integral being over any arc in $\mathbf{C} \backslash[-1,1]$. For a fixed $r(1 \leq r<N)$, Theorem A.1 applied to $F^{(-r)}$ gives

$$
F^{(-r)}(z)=\sum_{n=0}^{\infty} b_{n} Q_{n}(z)
$$

converging uniformly on any compact subset of $\mathbf{C} \backslash[-1,1]$, with

$$
b_{n}=\frac{2 n+1}{2 i \pi} \int_{\mathcal{C}} F^{(-r)}(z) P_{n}(z) d z \text {. }
$$

Here $\mathcal{C}$ may be $E(L)$ or $\{z:|z|=L\}$ for any $L>1$. Since $F^{(-r)}(z) \sim$ const. $z^{-N+r}$ at infinity, $b_{n}=0$ for $n<N-r-1$. We can now prove

Theorem A.3 Let $N$ and $r$ be integers such that $N-r-1 \geq r \geq 1$. Let $F$ be a function holomorphic in $\mathbf{C} \backslash[-1,1]$ and at infinity, with $c_{n}$ given by A.42) and $c_{n}=0$ for $n<N$. Then

$$
F(z)=\sum_{n=N-1}^{\infty} a_{n}\left(z^{2}-1\right)^{-r} D_{n}^{\frac{1}{2}-r}(z)
$$


with

$$
a_{n}=\frac{(2 n-2 r+1) \Gamma(n+1) \Gamma\left(\frac{1}{2}-r\right)^{2}}{2^{2 r+1} i \pi^{2} \Gamma(n-2 r+1)} \int_{\mathcal{C}} F(t) C_{n}^{\frac{1}{2}-r}(t) d t .
$$

The convergence is uniform on any compact subset of $\mathbf{C} \backslash[-1,1]$.

Proof. Eqs. (A.45) and (A.46) hold, and $b_{n}=0$ for $n<N-r-1$, in particular for $n<r$. Since the series in A.45 is a uniformly convergent series of holomorphic functions, it can be differentiated term by term:

$$
\begin{aligned}
F(z) & =\sum_{n=N-1-r}^{\infty} b_{n} Q_{n}^{(r)}(z) \\
& =\sum_{n=N-1}^{\infty} b_{n-r}\left(z^{2}-1\right)^{-r / 2} Q_{n-r}^{r}(z) \\
& =\sum_{n=N-1}^{\infty} b_{n-r} \frac{(-1)^{r} \Gamma(n+1) \Gamma\left(\frac{1}{2}-r\right)}{2^{r} \pi^{1 / 2} \Gamma(n-2 r+1)}\left(z^{2}-1\right)^{-r} D_{n}^{\frac{1}{2}-r}(z)
\end{aligned}
$$

In (A.46), we can substitute (see [15, 3.6.1 (8) p. 149 and 3.3 .1 (7) p. 140])

$$
\begin{aligned}
P_{n}(z) & =\left(\frac{d}{d z}\right)^{r}\left(z^{2}-1\right)^{r / 2} P_{n}^{-r}(z) \\
& =\left(\frac{d}{d z}\right)^{r} \frac{\Gamma(n-r+1)}{\Gamma(n+r+1)}\left(z^{2}-1\right)^{r / 2} P_{n}^{r}(z) \quad \text { provided } n \geq r .
\end{aligned}
$$

Therefore

$$
b_{n}=\frac{(-1)^{r}(2 n+1)}{2 i \pi} \int_{\mathcal{C}} F(t)\left(t^{2}-1\right)^{r / 2} P_{n}^{-r}(t) d t
$$

and for $n \geq 2 r$,

$$
\begin{aligned}
b_{n-r} & =\frac{(-1)^{r}(2 n-2 r+1) \Gamma(n-2 r+1)}{2 i \pi \Gamma(n+1)} \int_{\mathcal{C}} F(t)\left(t^{2}-1\right)^{r / 2} P_{n-r}^{r}(t) d t \\
& =\frac{(-1)^{r}(2 n-2 r+1) \Gamma\left(\frac{1}{2}-r\right)}{2^{r+1} i \pi^{3 / 2}} \int_{\mathcal{C}} F(t) C_{n}^{\frac{1}{2}-r}(t) d t .
\end{aligned}
$$

Substituting this into A.50 gives A.48 and proves the theorem.

\section{A.5 Expansion of $\mathbf{Q}_{n-r}^{r}$ in terms of the $\mathbf{P}_{k}^{r}$}

Recall that $P_{\nu}^{0}=P_{\nu}$ and $Q_{\nu}^{0}=Q_{\nu}$ are the Legendre functions of the first and second kind, and that, for integer $r \geq 1$, (see [15, 3.6.1 p.148-149]), for $-1<x<1$,

$$
\begin{aligned}
& \mathbf{P}_{\nu}^{r}(x)=(-1)^{r}\left(1-x^{2}\right)^{r / 2}\left(\frac{d}{d x}\right)^{r} \mathbf{P}_{\nu}(x), \\
& \mathbf{Q}_{\nu}^{r}(x)=(-1)^{r}\left(1-x^{2}\right)^{r / 2}\left(\frac{d}{d x}\right)^{r} \mathbf{Q}_{\nu}(x),
\end{aligned}
$$

The Legendre polynomials $P_{k}=\mathbf{P}_{k}$ form an orthogonal basis of $L^{2}([-1,1])$ (with the Lebesgue measure) and

$$
\int_{-1}^{1} \mathbf{P}_{k}(x) \mathbf{P}_{l}(x) d x=\frac{1}{k+\frac{1}{2}} \delta_{k l}
$$


so that, for any $f, g \in L^{2}([-1,1])$,

$$
\begin{aligned}
& \int_{-1}^{1} g(x) f(x) d x=\sum_{k=0}^{\infty} f_{k} \int_{-1}^{1} g(x) \mathbf{P}_{k}(x) d x \\
& f_{k}=\left(k+\frac{1}{2}\right) \int_{-1}^{1} f(x) \mathbf{P}_{k}(x) d x
\end{aligned}
$$

We may also regard $f$ and $\mathbf{P}_{k}$ as distributions. If $h$ is a $\mathcal{C}^{\infty}$ test-function with support contained in $(-1,1)$

$$
\begin{aligned}
& \int_{-1}^{1} h(x)(-1)^{r}\left(1-x^{2}\right)^{r / 2} f^{(r)}(x) d x \stackrel{\text { def }}{=} \int_{-1}^{1}\left(\left(\frac{d}{d x}\right)^{r}\left[h(x)\left(1-x^{2}\right)^{r / 2}\right]\right) f(x) d x \\
& =\sum_{k=0}^{\infty} f_{k} \int_{-1}^{1}\left(\left(\frac{d}{d x}\right)^{r}\left[h(x)\left(1-x^{2}\right)^{r / 2}\right]\right) \mathbf{P}_{k}(x) d x \\
& =\sum_{k=0}^{\infty} f_{k} \int_{-1}^{1} h(x) \mathbf{P}_{k}^{r}(x) d x .
\end{aligned}
$$

This will continue to hold if $h$ tends to a function such that

$$
x \mapsto\left(\frac{d}{d x}\right)^{r}\left[h(x)\left(1-x^{2}\right)^{r / 2}\right]
$$

defines an element of $L^{2}(\mathbf{R})$ with support in $[-1,1]$ ). We may in particular choose $f=\mathbf{Q}_{N}$, with $N \geq r \geq 1$. Then $f \in L^{2}([-1,1])$, and

$$
f_{k}=\left(k+\frac{1}{2}\right) \int_{-1}^{1} \mathbf{Q}_{N}(x) \mathbf{P}_{k}(x) d x=\left\{\begin{array}{l}
\frac{\left(1-(-1)^{k-N}\right)\left(k+\frac{1}{2}\right)}{(k-N)(k+N+1)} \quad \text { if } k \neq N \\
0 \quad \text { if } k=N
\end{array}\right.
$$

Thus

$$
\int_{-1}^{1} h(x) \mathbf{Q}_{N}^{r}(x) d x=\sum_{k=0}^{\infty} f_{k} \int_{-1}^{1} h(x) \mathbf{P}_{k}^{r}(x) d x,
$$

with the $f_{k}$ given by (A.63). Setting $N=n-r$, with an integer $n \geq 2 r$, we obtain

$$
\int_{-1}^{1} h(x) \mathbf{Q}_{n-r}^{r}(x) d x=\sum_{k=2 r}^{\infty} a_{r}(k, n) \int_{-1}^{1} h(x) \mathbf{P}_{k-r}^{r}(x) d x,
$$

with

$$
a_{r}(k, n)=a(k-r, n-r), \quad a(k, n)= \begin{cases}\frac{1}{k-n}+\frac{1}{k+n+1} & \text { if } k-n \text { is odd } \\ 0 & \text { if } k-n \text { is even }\end{cases}
$$

\section{B Appendix. Extension of Hsü's Theorem}

Theorem B.1 (Hsü [16]) Let $r$ be complex with $\operatorname{Re} r<1$, and $n_{1}, n_{2}, n_{3}$ be non-negative integers. Then the integral

$$
\int_{-1}^{1}\left(1-x^{2}\right)^{-r} C_{n_{1}}^{\frac{1}{2}-r}(x) C_{n_{2}}^{\frac{1}{2}-r}(x) C_{n_{3}}^{\frac{1}{2}-r}(x) d x
$$


vanishes unless

$$
n_{j} \leq n_{k}+n_{\ell}, \quad 2 s \stackrel{\text { def }}{=} n_{1}+n_{2}+n_{3} \text { is even }
$$

for every permutation $(j, k, \ell)$ of $(1,2,3)$. If the above conditions are satisfied,

$$
\begin{aligned}
& \int_{-1}^{1}\left(1-x^{2}\right)^{-r} C_{n_{1}}^{\frac{1}{2}-r}(x) C_{n_{2}}^{\frac{1}{2}-r}(x) C_{n_{3}}^{\frac{1}{2}-r}(x) d x= \\
& =2^{r} \pi \frac{\Gamma(s+1-2 r)}{\Gamma\left(s+\frac{3}{2}-r\right) \Gamma\left(\frac{1}{2}-r\right)} \times \\
& \times \frac{\Gamma\left(s-n_{1}+\frac{1}{2}-r\right)}{\Gamma\left(s-n_{1}+1\right) \Gamma\left(\frac{1}{2}-r\right)} \frac{\Gamma\left(s-n_{2}+\frac{1}{2}-r\right)}{\Gamma\left(s-n_{2}+1\right) \Gamma\left(\frac{1}{2}-r\right)} \frac{\Gamma\left(s-n_{3}+\frac{1}{2}-r\right)}{\Gamma\left(s-n_{3}+1\right) \Gamma\left(\frac{1}{2}-r\right)} \\
& \stackrel{\text { def }}{=} H\left(r ; n_{1}, n_{2}, n_{3}\right) .
\end{aligned}
$$

In the sequel we will take $H\left(r ; n_{1}, n_{2}, n_{3}\right)$ to be defined by the meromorphic function of $r$ appearing in the rhs of (B.3) if the conditions (B.2) hold, and by 0 otherwise. We abbreviate $H\left(r ; n_{1}, n_{2}, n_{3}\right)$ to $H(r)$ when no ambiguity arises.

Remark B.1 It is important to note that if $n_{1}, n_{2}, n_{3}$ are fixed non-negative integers, then $r \mapsto$ $H\left(r ; n_{1}, n_{2}, n_{3}\right)$ is holomorphic at every integer value of $r$ such that $n_{j}-2 r \geq 0$ for at least two distinct values of $j=1,2,3$. This is obvious if the conditions (B.2) are not satisfied since $H\left(r ; n_{1}, n_{2}, n_{3}\right)=0$ in this case. If the conditions (B.2) are satisfied, the three last factors in the rhs of (B.3) are polynomials in $r$, while the argument of the first Gamma function is $\geq 1$.

\section{B.1 Contour integrals}

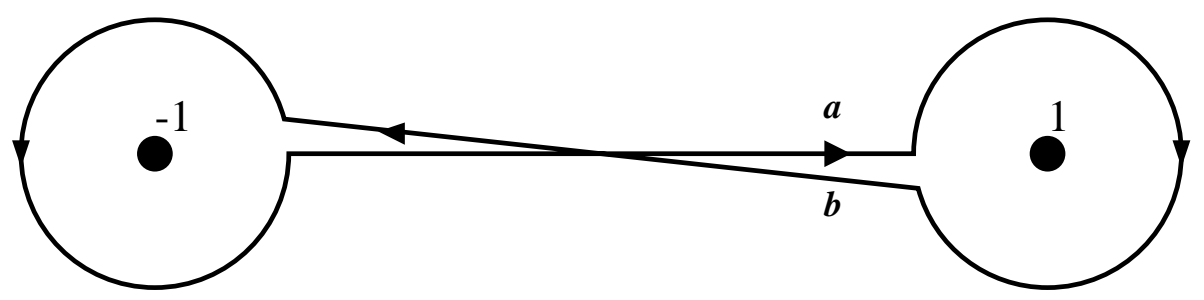

Figure 1: The contour $\mathcal{C}_{1}$

Let $\mathcal{C}_{1}$ be the contour in Fig. 1, which is homotopic to a figure eight. The radii of the two circles are to be regarded as small and the two straight segments are very close to the real axis. Let $\Phi(x, r)$ be an entire function of $x$ and $r$. Let

$$
K(r)=\int_{\mathcal{C}_{1}}\left(1-x^{2}\right)^{-r} \Phi(x, r) d x
$$

To make things more definite, we assume that the segment $a$ lies on the real axis inside the open interval $(-1,1)$, and that on this segment $\left(1-x^{2}\right)^{-r}=\left|1-x^{2}\right|^{-r}$. Then the contour may be considered as a closed curve in the Riemann surface of $z \mapsto\left(1-z^{2}\right)^{-r} \Phi(z, r)$. The function $K$ is entire and can, of course, be defined with any smooth closed contour homotopic to $\mathcal{C}_{1}$ in that Riemann surface.

Let first $\operatorname{Re} r<1$. Then the integral

$$
I(r)=\int_{-1}^{1}\left(1-x^{2}\right)^{-r} \Phi(x, r) d x
$$


exists and is holomorphic in $r$. If the radii of the circles (centered at 1 and -1 ) which appear in $\mathcal{C}_{1}$ tend to 0 , their contributions to $K(r)$ tend to 0 , and the contributions of the two segments become

$$
\begin{aligned}
& a: I(r) \\
& b:-e^{-2 i \pi r} I(r),
\end{aligned}
$$

so that, for $\operatorname{Re} r<1$,

$$
K(r)=\left(1-e^{-2 i \pi r}\right) I(r)=2 i e^{-i \pi r} \sin (\pi r) I(r) .
$$

This holomorphic function of $r$ vanishes at every integer value of $r$ in the half-plane $r<1$.

In the case of Hsü's integral,

$$
\Phi(x, r)=C_{n_{1}}^{\frac{1}{2}-r}(x) C_{n_{2}}^{\frac{1}{2}-r}(x) C_{n_{3}}^{\frac{1}{2}-r}(x),
$$

where the $n_{j}$ are non-negative integers. For $\operatorname{Re} r<1, I(r)$ is given by Theorem B, i.e. $I(r)=H(r)$, where $H$ is the explicit meromorphic function of $r$ (identically 0 if the conditions (B.2) are not satisfied) defined in the preceding subsection. Therefore, by analytic continuation,

$$
K(r) \stackrel{\text { def }}{=} \int_{\mathcal{C}_{1}}\left(1-x^{2}\right)^{-r} \Phi(x, r) d x=\left(1-e^{-2 i \pi r}\right) H(r)=2 i e^{-i \pi r} \sin (\pi r) H(r)
$$

and

$$
K^{\prime}(r)=2 i \pi e^{-2 i \pi r} H(r)+\left(1-e^{-2 i \pi r}\right) H^{\prime}(r) .
$$

hold for all $r$ at which $H$ is regular. In particular, for any integer $p$ such that $n_{j}-2 r \geq 0$ for at least two distinct $j$ (see Remark B.1 above),

$$
K(p)=0, \quad K^{\prime}(p)=2 i \pi H(p) .
$$

On the other hand

$$
K^{\prime}(r)=\int_{\mathcal{C}_{1}}\left[-\left(1-x^{2}\right)^{-r} \log \left(1-x^{2}\right) \Phi(x, r)+\left(1-x^{2}\right)^{-r} \frac{\partial}{\partial r} \Phi(x, r)\right] d x .
$$

If $\Phi(x, r$ is given by (B.9),

$$
\frac{\partial}{\partial r} \Phi(x, r)=\sum_{\pi}\left((\partial / \partial r) C_{n_{j}}^{\frac{1}{2}-r}(x)\right) C_{n_{k}}^{\frac{1}{2}-r}(x) C_{n_{l}}^{\frac{1}{2}-r}(x),
$$

where the sum is over the cyclic permutations $(j, k, l)$ of $(1,2,3)$. Let $p$ be a non-negative integer such that two of the inequalities $n_{1}-2 p \geq 0, n_{2}-2 p \geq 0, n_{3}-2 p \geq 0$, hold. Then (see (B.14) $) \Phi(x, p)$ and $(\partial / \partial r) \Phi(x, p)$ are polynomials in $x$ divisible by $\left(1-x^{2}\right)^{p}$. Therefore the integrands of (B.10) and (B.13) become, for $r=p$, integrable on $[-1,1]$, and $K^{\prime}(r)$ can be expressed in terms of the integral of its integrand on $[-1,1]$. The contributions of the two segments of the contour are :

$$
\begin{aligned}
& a: \int_{-1}^{1}\left[-\left(1-x^{2}\right)^{-p} \log \left(1-x^{2}\right) \Phi(x, p)+\left(1-x^{2}\right)^{-p} \frac{\partial}{\partial r} \Phi(x, p)\right] d x \\
& b:-\int_{-1}^{1}\left[-\left(1-x^{2}\right)^{-p}\left[\log \left(1-x^{2}\right)+2 i \pi\right] \Phi(x, p)+\left(1-x^{2}\right)^{-p} \frac{\partial}{\partial r} \Phi(x, p)\right] d x .
\end{aligned}
$$

so that

$$
K^{\prime}(p)=2 i \pi \int_{-1}^{1}\left(1-x^{2}\right)^{-p} \Phi(x, p) d x
$$

and hence

$$
\int_{-1}^{1}\left(1-x^{2}\right)^{-p} \Phi(x, p) d x=H(p)
$$




\section{B.2 Extension of Hsü's Theorem}

We have thus obtained the following extension of Hsü's Theorem:

Lemma B.1 Let $n_{1}, n_{2}, n_{3}$ be non-negative integers and $r \in \mathbf{C}$ satisfy one of the two following conditions:

(i) $\operatorname{Re} r<1$,

(ii) $r$ is an integer and $n_{j}-2 r \geq 0$ for at least two distinct values of $j \in\{1,2,3\}$.

Then the statement of Theorem B.1 holds, i.e. i.e. the integral in the lhs of Eq. (B.3) exists and it is equal to $H\left(r ; n_{1}, n_{2}, n_{3}\right)$, i.e. the expression (B.3) if the conditions (B.2) are satisfied and 0 otherwise.

The above phenomenon also occurs if $\Phi(x, r)$ is a product of two Gegenbauer polynomials instead of three. In this case the role of Hsü's formula is played by the orthogonality relation (see [15, 3.15.1 p. $177])$ :

$$
\int_{-1}^{1}\left(1-x^{2}\right)^{-r} C_{n}^{\frac{1}{2}-r}(x) C_{m}^{\frac{1}{2}-r}(x) d x=\frac{2^{2 r} \pi \Gamma(n+1-2 r)}{\left(n+\frac{1}{2}-r\right) \Gamma\left(\frac{1}{2}-r\right)^{2} \Gamma(n+1)} \delta_{n m}, \quad \operatorname{Re} r<1 .
$$

The preceding argument, with $\Phi(x, r)=C_{n}^{\frac{1}{2}-r}(x) C_{m}^{\frac{1}{2}-r}(x)$, shows that (B.18) still holds for positive integer $r$ such that $n-2 r \geq 0, m-2 r \geq 0$.

\section{Appendix. An identity for hypergeometric functions}

The identity [15, 2.1.5 (26), p. 65] can be rewritten as

$$
\begin{aligned}
& F\left(a, a+\frac{1}{2} ; c ; u\right)=(v+1)^{2 a} F(2 a, 2 a-c+1 ; c ; v) \\
& v=\frac{1-(1-u)^{\frac{1}{2}}}{1+(1-u)^{\frac{1}{2}}}, \quad u=\frac{4 v}{(v+1)^{2}}
\end{aligned}
$$

Letting $z=\frac{1}{2}\left(\zeta+\zeta^{-1}\right), u=z^{-2}$ and $v=\zeta^{-2}$ implies that (C.2 holds, and we obtain the identity

$$
\begin{aligned}
& F\left(a, a+\frac{1}{2} ; c ; \frac{1}{z^{2}}\right)=\left(1+\frac{1}{\zeta^{2}}\right)^{2 a} F\left(2 a, 2 a-c+1 ; c ; \frac{1}{\zeta^{2}}\right) \\
& \zeta=z+\left(z^{2}-1\right)^{\frac{1}{2}}, \quad \zeta^{-1}=z-\left(z^{2}-1\right)^{\frac{1}{2}}, \quad z=\frac{\zeta+\zeta^{-1}}{2} .
\end{aligned}
$$

Note that (C.1) and (C.3) can be respectively rewritten as

$$
u^{a} F\left(a, a+\frac{1}{2} ; c ; u\right)=(4 v)^{a} F(2 a, 2 a-c+1 ; c ; v)
$$

and

$$
(2 z)^{-2 a} F\left(a, a+\frac{1}{2} ; c ; \frac{1}{z^{2}}\right)=\zeta^{-2 a} F\left(2 a, 2 a-c+1 ; c ; \frac{1}{\zeta^{2}}\right)
$$

\section{Appendix. Proof of the projector identity (Theorem 8.1)}

In this appendix, $d \geq 2$ is always an integer, and $\lambda=(d-1) / 2$. Hence, for integer $n>-2 \lambda$, $D_{n}^{\lambda}$ is holomorphic in $\widehat{\Delta}_{1}$. We will use a very crude bound on $\left|D_{n}^{\lambda}(z)\right|$ which is valid if $n$ is real (not necessarily integer or positive), and $n+2 \lambda>0, z \notin[-1,1]$ :

$$
\left|D_{n}^{\lambda}(z)\right| \leq \text { Const. }|z|^{-n-2 \lambda}\left(1+\operatorname{dist.}(z,[-1,1])^{-1}\right)^{2 \lambda}
$$


The constant depends on $n$ and $\lambda$. This is easily derived from (2.14).

It is proved in [6] that the tuboid $\mathcal{T}_{1+}$ (defined in (2.3) $)$ is the set of points $w=\Lambda z$ where $\Lambda \in G_{0}$ and $z$ is of the special form

$$
z=\exp \left(i \alpha M_{0 d}\right) e_{d}=(\sin (i \alpha), \overrightarrow{0}, \cos (i \alpha)), \quad \alpha>0
$$

If $z \in \mathcal{T}_{1 \pm}$ and $u \in X_{d}$ then $z \cdot u \in \mathbf{C} \backslash[-1,1]$ so that, for integer $n, u \mapsto D_{n}^{\lambda}(z \cdot u)$ is $\mathcal{C}^{\infty}$ (actually analytic). We will prove

Lemma D.1 Let $n_{1}, n_{2}$ and $d$ be integers satisfying $d \geq 2, n_{1}+d-1>0, n_{2}+d-1>0$ and $n_{1}+n_{2}+d-1>0$. Let $z_{1} \in \mathcal{T}_{1-}$ and $z_{2} \in \mathcal{T}_{1+}$. Then the integral

$$
I_{n_{1}, n_{2}, \frac{d-1}{2}}\left(z_{1}, z_{2}\right)=\int_{X_{d}} D_{n_{1}^{2}}^{\frac{d-1}{2}}\left(z_{1} \cdot u\right) D_{n_{2}^{2}}^{\frac{d-1}{2}}\left(u \cdot z_{2}\right) d u
$$

is absolutely convergent and

$$
I_{n_{1}, n_{2}, \frac{d-1}{2}}\left(z_{1}, z_{2}\right)=\delta_{n_{1} n_{2}} C\left(n_{1}, d\right) D_{n_{1}^{\frac{d-1}{2}}}\left(z_{1} \cdot z_{2}\right)
$$

with

$$
C(n, d)=\frac{4 \pi^{\frac{d+3}{2}}}{(2 n+d-1) \Gamma\left(\frac{d-1}{2}\right)} .
$$

Here $d u$ denotes the standard invariant measure on $X_{d}$, i.e. $d u=2 \delta(u \cdot u-1) d u^{0} \ldots d u^{d}$. The convergence takes place in the space of functions with tempered behavior in $\mathcal{T}_{1-} \times \mathcal{T}_{1+}$.

Note that if $n_{1}=n_{2}=n$, the condition $2 n+d-1>0$ must be satisfied.

Proof. Let $n$ and $d$ be integers such that $d \geq 2$ and $n+d-1>0$ and $p \in \mathbf{R}$ satisfy $p(n+d-1)-d+1>0$, i.e.

$$
0<\frac{1}{p}<\frac{n+d-1}{d-1} .
$$

We will verify that, for $z=x+i y \in \mathcal{T}_{+}$(and similarly for $z \in \mathcal{T}_{-}$), the integral

$$
J_{n, p}(z)=\int_{X_{d}}\left|D_{n}^{\frac{d-1}{2}}(z \cdot u)\right|^{p} d u
$$

is absolutely convergent. By a transformation in $G_{0}$ we can bring $z$ to the form (D.2). Taking $u$ in the form

$$
u=(s \sin \theta, r \vec{v}, s \cos \theta)
$$

with $\vec{v}$ a $(d-1)$-dimensional unit vector, we find after integrating over $\vec{v}$

$$
\begin{aligned}
J_{n, p}(z) & =2 \Omega_{d-1} \int_{0}^{\infty} s d s \int_{0}^{\infty} r^{d-2} d r \int_{0}^{2 \pi} d \theta \delta\left(s^{2}-r^{2}-1\right)\left|D_{n}^{\frac{d-1}{2}}(s \cos (\theta-i \alpha))\right|^{p} \\
& =\Omega_{d-1} \int_{0}^{\infty} r^{d-2} d r \int_{0}^{2 \pi}\left|D_{n^{\frac{d-1}{2}}}\left(\sqrt{r^{2}+1} \cos (\theta-i \alpha)\right)\right|^{p} d \theta, \quad \Omega_{d-1}=\frac{2 \pi^{\frac{d-1}{2}}}{\Gamma\left(\frac{d-1}{2}\right)} .
\end{aligned}
$$

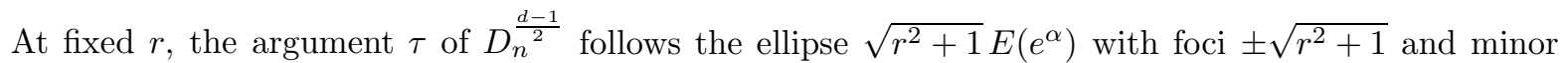
semi-axis $\sqrt{r^{2}+1} \operatorname{sh}(\alpha)$. Hence $|\tau| \geq \sqrt{r^{2}+1} \operatorname{sh}(\alpha)$ and dist. $(\tau,[-1,1]) \geq \sqrt{r^{2}+1}(\operatorname{ch}(\alpha)-1)$. The full integrand is thus majorized by const. $(\operatorname{sh} \alpha)^{-p(n+d-1)} r^{-p(n+d-1)+d-2}\left(1+\operatorname{sh}(\alpha / 2)^{-2}\right)^{p d}$, and the integral is absolutely convergent. Since $y \cdot y=\operatorname{sh}(\alpha)^{2}$, the convergence takes place in the space of functions of $z$ bounded in modulus by a fixed negative power of $y \cdot y$.

The condition $n_{1}+n_{2}+d-1>0$ postulated in the lemma can be rewritten as

$$
1<\frac{n_{1}+d-1}{d-1}+\frac{n_{2}+d-1}{d-1} .
$$


If it is satisfied, it is possible to find $p_{1}>0, p_{2}>0$ such that

$$
\frac{1}{p_{1}}+\frac{1}{p_{2}}=1, \quad \frac{1}{p_{1}}<\frac{n_{1}+d-1}{d-1}, \quad \frac{1}{p_{2}}<\frac{n_{2}+d-1}{d-1} .
$$

By Hölder's inequality,

$$
\left|I_{n_{1}, n_{2}, \frac{d-1}{2}}\left(z_{1}, z_{2}\right)\right| \leq\left(J_{n_{1}, p_{1}}\left(z_{1}\right)\right)^{\frac{1}{p_{1}}}\left(J_{n_{2}, p_{2}}\left(z_{2}\right)\right)^{\frac{1}{p_{2}}}
$$

in which each of the integrals in the rhs is absolutely convergent. Again the convergence takes place in the space of functions with tempered behavior in $\mathcal{T}_{1-} \times \mathcal{T}_{1+}$. Since the convergence is in particular uniform on compact subsets of $\mathcal{T}_{1-} \times \mathcal{T}_{1+}$, the result $I_{n_{1}, n_{2}, \frac{d-1}{2}}\left(z_{1}, z_{2}\right)$ is an invariant function of $z_{1}$ and $z_{2}$, holomorphic in $\mathcal{T}_{1-} \times \mathcal{T}_{1+}$. It is therefore equal to a function of $z_{1} \cdot z_{2}$ holomorphic in $\Delta_{1}$ and tending to 0 at infinity, thus holomorphic in $\widehat{\Delta}_{1}$. It satisfies, in each of the two variables, the Klein-Gordon equation with square masses $n_{1}\left(n_{1}+d-1\right)$ and $n_{2}\left(n_{2}+d-1\right)$ respectively. It must therefore vanish if $n_{1} \neq n_{2}$. If $n_{1}=n_{2}$, these properties characterize $w_{n_{1}+\frac{d-1}{2}}\left(z_{1} \cdot z_{2}\right)$ up to a constant factor. It follows that (D.4) holds. In order to compute the constant $C(n, d)$, we specialize to the case $n_{2}=n_{1}=n$ and $z_{1}^{*}=z_{2}=z, z$ of the form (D.2). We find

$$
\Omega_{d-1} \int_{0}^{\infty} r^{d-2} d r \int_{0}^{2 \pi}\left|D_{n^{\frac{d-1}{2}}}\left(\sqrt{r^{2}+1} \cos (\theta-i \alpha)\right)\right|^{2} d \theta=C(n, d) D_{n^{\frac{d-1}{2}}}(\operatorname{ch}(2 \alpha)) .
$$

Setting $\zeta=e^{\alpha}$, both sides are analytic in $\zeta$ in a neighborhood of infinity and have a convergent expression in $1 / \zeta$. Identifiying the first terms of the expansions of both sides will give $C(n, d)$ :

$$
\begin{gathered}
\zeta^{-2 n-2 d+2} \frac{\Omega_{d-1} 2 \pi^{2} \Gamma(n+d-1)}{\Gamma\left(\frac{d-1}{2}\right) \Gamma\left(n+\frac{d+1}{2}\right)} \int_{0}^{\infty} r^{d-2}\left(r^{2}+1\right)^{-n-d+1} d r=C(n, d) \zeta^{-2 n-2 d+2} \\
C(n, d)=\frac{\Omega_{d-1} 2 \pi^{2} \Gamma(n+d-1)}{\Gamma\left(\frac{d-1}{2}\right) \Gamma\left(n+\frac{d+1}{2}\right)} \int_{0}^{\infty} r^{d-2}\left(r^{2}+1\right)^{-n-d+1} d r .
\end{gathered}
$$

The last integral is equal to

$$
\begin{aligned}
\frac{1}{2} \int_{0}^{\infty} t^{\frac{d-3}{2}}(t+1)^{-n-d+1} d t & =\frac{1}{2} \mathrm{~B}\left(\frac{d-1}{2}, n+\frac{d-1}{2}\right) \\
& =\frac{\Gamma\left(\frac{d-1}{2}\right) \Gamma\left(n+\frac{d-1}{2}\right)}{2 \Gamma(n+d-1)}
\end{aligned}
$$

([15, p. 9]). Hence

$$
C(n, d)=\frac{4 \pi^{\frac{d+3}{2}}}{(2 n+d-1) \Gamma\left(\frac{d-1}{2}\right)} .
$$

This completes the proof of the lemma.

We may reexpress this in terms of $w_{\nu}, \nu=n+(d-1) / 2$, given by (2.12):

$$
\int_{X_{d}} w_{n_{1}+\frac{d-1}{2}}\left(z_{1} \cdot u\right) w_{n_{2}+\frac{d-1}{2}}\left(u \cdot z_{2}\right) d u=\frac{2 \pi}{\left(2 n_{1}+d-1\right)} \delta_{n_{1} n_{2}} w_{n_{1}+\frac{d-1}{2}}\left(z_{1} \cdot z_{2}\right) .
$$

This proves Theorem 8.1 .

\section{References}

[1] J. M. Maldacena, "The Large N limit of superconformal field theories and supergravity," Adv. Theor. Math. Phys. 2, 231 (1998) [Int. J. Theor. Phys. 38, 1113 (1999)] arXiv:hep-th/9711200. 
[2] C. G. . Callan and F. Wilczek, "Infrared behavior at negative curvature," Nucl. Phys. B 340, 366 (1990).

[3] J. Bros, H. Epstein, M. Gaudin, U. Moschella and V. Pasquier, "Triangular invariants, three-point functions and particle stability on the de Sitter universe," Commun. Math. Phys. 295, 261 (2010) arXiv:0901.4223 [hep-th]].

[4] J. Bros, H. Epstein and U. Moschella, "Particle decays and stability on the de Sitter universe," Annales Henri Poincare 11, 611 (2010) arXiv:0812.3513 [hep-th]].

[5] J. Bros, H. Epstein and U. Moschella, "Lifetime of a massive particle in a de Sitter universe," JCAP 0802, 003 (2008) arXiv:hep-th/0612184.

[6] J. Bros, H. Epstein and U. Moschella, "Towards a general theory of quantized fields on the anti-de Sitter space-time," Commun. Math. Phys. 231, 481 (2002) arXiv:hep-th/0111255.

[7] R. F. Streater and A. S. Wightman: PCT, Spin and Statistics, and All That, Benjamin, New York. Princeton, (1964).

[8] D. W. Dusedau and D. Z. Freedman, "Lehmann spectral representation for anti-de Sitter quantum field theory," Phys. Rev. D 33 (1986) 389.

[9] D. W. Dusedau and D. Z. Freedman, "Renormalization in anti-de Sitter supersymmetry," Phys. Rev. D 33, 395 (1986).

[10] D. Kabat, G. Lifschytz and D. A. Lowe, "Constructing local bulk observables in interacting AdS/CFT," Phys. Rev. D 83, 106009 (2011) [arXiv:1102.2910 [hep-th]].

[11] J. Bouttier and E. Guitter, "Planar maps and continued fractions," arXiv:1007.0419v1 [math.CO].

[12] R. Askey. Orthogonal Polynomials and Special Functions Society For Industrial And Applied Mathematics, Philadelphia, PA, 1975

[13] G, E. Andrews, R. Askey, and R. Roy. Special Functions Cambridge University Press, Cambridge 1999

[14] N.N. Bogolubov, A.A. Logunov, A.I. Oksak, and I.T. Todorov. General principles of Quantum Field Theory. Springer-Verlag, Berlin Heidelberg New York, 1990.

[15] A. Erdélyi. The Bateman manuscript project. Higher Transcendental Functions, volume I. McGrawHill, New York, 1953.

[16] H. Hsü. "Certain integrals and infinite series involving ultraspherical polynomials and Bessel functions," Duke Mathematical Journal, 4, 374-383 (1938)

[17] G. Szegö. Orthogonal Polynomials. Amer. Math. Soc, Providence, RI, 1975. 